\title{
IMPDH inhibitors for antitumor therapy in tuberous sclerosis complex
}

\author{
Alexander J. Valvezan, ${ }^{1}$ Molly C. McNamara, ${ }^{1}$ Spencer K. Miller, ${ }^{1}$ Margaret E. Torrence, ${ }^{1}$ \\ John M. Asara, ${ }^{2}$ Elizabeth P. Henske, ${ }^{3}$ and Brendan D. Manning ${ }^{1}$ \\ 'Department of Molecular Metabolism, Harvard T.H. Chan School of Public Health, Boston, Massachusetts, USA. \\ ${ }^{2}$ Division of Signal Transduction, Beth Israel Deaconess Medical Center, and Department of Medicine, Harvard Medical \\ School, Boston, Massachusetts, USA. ${ }^{3}$ Department of Medicine, Brigham and Women's Hospital and Harvard Medical \\ School, Boston, Massachusetts, USA.
}

Recent studies in distinct preclinical tumor models have established the nucleotide synthesis enzyme inosine-5'-monophosphate dehydrogenase (IMPDH) as a viable target for antitumor therapy. IMPDH inhibitors have been used clinically for decades as safe and effective immunosuppressants. However, the potential to repurpose these pharmacological agents for antitumor therapy requires further investigation, including direct comparisons of available compounds. Therefore, we tested structurally distinct IMPDH inhibitors in multiple cell and mouse tumor models of the genetic tumor syndrome tuberous sclerosis complex (TSC). TSC-associated tumors are driven by uncontrolled activation of the growth-promoting protein kinase complex mechanistic target of rapamycin (mTOR) complex 1 (mTORC1), which is also aberrantly activated in the majority of sporadic cancers. Despite eliciting similar immunosuppressive effects, the IMPDH inhibitor mizoribine, used clinically throughout Asia, demonstrated far superior antitumor activity compared with the FDA-approved IMPDH inhibitor mycophenolate mofetil (or CellCept, a prodrug of mycophenolic acid). When compared directly to the mTOR inhibitor rapamycin, mizoribine treatment provided a more durable antitumor response associated with tumor cell death. These results provide preclinical support for repurposing mizoribine, over other IMPDH inhibitors, as an alternative to mTOR inhibitors for the treatment of TSC-associated tumors and possibly other tumors featuring uncontrolled mTORC1 activity.

Conflict of interest: BDM is a shareholder and scientific advisory board member for Navitor Pharmaceuticals and LAM Therapeutics.

Copyright: () 2020, American Society for Clinical Investigation.

Submitted: November 18, 2019

Accepted: March 5, 2020

Published: April 9, 2020

Reference information: /CI Insight. 2020;5(7):e135071.

https://doi.org/10.1172/jci.

insight.135071.

\section{Introduction}

Tumor cells must synthesize new biomass to support their uncontrolled growth and proliferation. This is often achieved, in part, through activation of the mechanistic target of rapamycin complex 1 (mTORC1), a central regulator of anabolic cell growth that is active in the majority of human cancers, across nearly all lineages $(1,2)$. mTORC1 induces a coordinated anabolic program to stimulate synthesis of the major macromolecules required for cell growth, including proteins, lipids, and nucleic acids (3). mTORC1 also stimulates flux through metabolic pathways that support macromolecule synthesis, including the de novo purine and pyrimidine nucleotide synthesis pathways, glycolysis, and the oxidative branch of the pentose phosphate pathway (4-7). Given its role in tumor cell growth and metabolism, there has been much interest in targeting mTORC1 in cancer. However, with over 1000 clinical trials to date, FDA-approved mTORC1 inhibitors (rapamycin/sirolimus and its analogs) have shown limited efficacy as single-agent therapies, consistent with their established growth-slowing, cytostatic effects rather than the cytotoxic effects needed to eradicate tumor cells (8).

Rapamycin and its analogs are currently in frequent use for treatment of tumors in tuberous sclerosis complex (TSC), a genetic syndrome caused by loss-of-function mutations in the TSC1 or TSC2 tumor suppressor genes, which encode the essential components of the TSC protein complex (TSC complex) (9). The TSC complex inhibits the Ras-related GTPase Rheb, which is an essential upstream activator of mTORC1; thus tumors in patients with TSC are driven by robust, uncontrolled mTORC1 activity (10). TSC is a pleiotropic disorder in which patients commonly develop neurological phenotypes, including epilepsy, autism, and a variety of cognitive and behavioral manifestations (collectively referred to as TSC-associated neuropsychiatric disorders), accompanied by widespread tumor development across 
multiple organ systems, including, but not limited to, the brain (tubers and subependymal giant cell astrocytomas), heart (rhabdomyomas), kidney (angiomyolipomas), skin (fibromas), and lung (lymphangioleiomyomatosis, LAM) (11). LAM is a proliferative and destructive lung disorder that can lead to respiratory failure, is nearly exclusive to women, and arises both in TSC patients and sporadically through inactivating mutations in TSC1 or TSC2 (12). Rapamycin and its analogs can slow or shrink tumors in TSC and LAM, but tumors are not eliminated by these agents and can rapidly regrow when treatment is discontinued $(13,14)$. Loss-of-function mutations in TSC1 and TSC2 are also found in sporadic cancers, with the highest frequency being in bladder cancer and hepatocellular carcinoma $(15,16)$. Thus, there is an unmet clinical need to selectively induce cell death in TSC1/2-deficient tumors. Finally, it is worth noting that the primary route to uncontrolled mTORC1 activity in human cancers is through aberrant inhibition of the TSC complex because some of the most commonly altered oncogenes (e.g., $P I K 3 C A, K R A S)$ and tumor suppressors (PTEN, NF1, LKB1) lie within signaling pathways that converge on regulation of the TSC complex to control mTORC1 (17).

As an alternative to inhibiting mTORC1 itself, it might be possible to elicit sustained antitumor responses by targeting specific processes within the downstream metabolic network activated as part of the cell growth program of mTORC1. Indeed, we previously demonstrated that aberrant mTORC1 activation increases dependence on guanylate nucleotide synthesis for cell viability. In cells and tumors with active mTORC1, we found that guanylate synthesis is required to meet the increased demand for nucleotides that comes from mTORC1 stimulating ribosomal RNA synthesis (18), exemplifying the importance of mTORC1 coupling distinct metabolic pathways within the broader anabolic program it induces. This metabolic vulnerability can be exploited by inhibiting inosine-5'-monophosphate dehydrogenase (IMPDH), the rate-limiting enzyme in de novo guanylate nucleotide synthesis. IMPDH inhibition was found to rapidly deplete guanylates in cells and tumors with active mTORC1, resulting in selective induction of sustained DNA replication stress that culminates in DNA damage and apoptosis (18). Thus, in contrast to mTORC1 inhibitors, IMPDH inhibition is selectively cytotoxic to cells and tumors with uncontrolled mTORC1 signaling. Importantly, inhibition of mTORC1 with rapamycin protects TSC2-deficient cells from the apoptosis-inducing effects of IMPDH inhibitors. Inhibition of mTORC1 or its downstream synthesis of ribosomal RNA (rRNA) spares intracellular guanylates and counteracts the effects of IMPDH inhibitors by reducing the corresponding demand for nucleotides.

IMPDH2 is frequently upregulated in human cancers, including a subset of small-cell lung cancers where IMPDH inhibition was recently shown to have antitumor efficacy in preclinical tumor models (19). IMPDH inhibition has also shown efficacy in xenograft tumors established using human glioblastoma, leukemia, lymphoma, pancreatic adenocarcinoma, and colon adenocarcinoma cell lines (20-22). If these preclinical findings are to be translated into clinical antitumor therapy, it is essential to directly compare the efficacy of available IMPDH inhibitors.

Activated lymphocytes require mTORC1 signaling and de novo nucleotide synthesis pathways for their rapid expansion, and thus inhibitors of these pathways, including rapamycin and IMPDH inhibitors, are used clinically as immunosuppressants. IMPDH inhibitors are well tolerated, especially when compared with classical chemotherapeutic agents that directly target deoxyribonucleotide triphosphate or DNA synthesis (23), making them attractive for potential repurposing. Of the clinically approved IMPDH inhibitors, mizoribine (Bredinin) is a natural purine analog used predominantly in Asia for autoimmune disorders and preventing organ rejection after transplant; mycophenolic acid (MPA, Myfortic) and its orally bioavailable prodrug, mycophenolate mofetil (MMF, CellCept), are FDA approved and structurally distinct from mizoribine but used for similar indications; and ribavirin (Rebetol, Copegus) is an FDA-approved purine analog, which, unlike mizoribine, can incorporate into RNA and is used as an antiviral agent $(24,25)$. Here, we test and directly compare these and other IMPDH inhibitors, and a collection of available small-molecule inhibitors of nucleotide synthesis, for selective targeting of cells with active mTORC1 using multiple cell and tumor models of TSC.

\section{Results}

Mizoribine is the most selective nucleotide synthesis inhibitor for targeting TSC2-deficient cells in vitro. We previously demonstrated that uncontrolled mTORC1 activation stemming from loss of the TSC complex increases cellular dependence on guanylate synthesis for growth and viability and that inhibitors of IMPDH induce apoptosis selectively in cells with active mTORC1 (18). To determine whether other enzymatic targets in nucleotide synthesis pathways can elicit a similar preferential response, littermate-derived $T s c 2^{+/+}$and $\mathrm{Tsc}^{-1-} \mathrm{MEFs}$ were treated with a 
panel of available inhibitors of enzymes in the de novo purine and pyrimidine synthesis and salvage pathways (Supplemental Figure 1, A-G; supplemental material available online with this article; https://doi.org/10.1172/ jci.insight.135071DS1). However, none of these compounds preferentially inhibited the proliferation of $T s c 2^{-1-}$ cells compared with $T s c 2^{+/+}$, including inhibitors of dihydrofolate reductase (methotrexate), hypoxanthine-guanine phosphoribosyltransferase (6-mercaptopurine), dihydroorotate dehydrogenase (A771726 and brequinar), uridine monophosphate synthase (pyrazofurin), cytidine triphosphate synthase (3-deazauridine), and thymidylate synthase (5-fluorouracil). Thus, we focused on structurally distinct inhibitors of IMPDH to identify the compound that most selectively inhibits the viability of cells with uncontrolled mTORC1 signaling.

The clinically used compounds mizoribine, MPA, and ribavirin, which inhibit both IMPDH isoforms (IMPDH1 and IMPDH2), all preferentially reduced the proliferation of $T s c 2^{-1-}$ cells compared with $T s c 2^{+/+}$, with mizoribine exhibiting the most selective effects on $T s c 2^{-/-}$cells and ribavirin the least (Figure 1A). At effective doses, MPA and ribavirin were more generally cytotoxic to both wild-type and $T s c 2^{-1-}$ cells relative to mizoribine. A fourth IMPDH inhibitor that is not in clinical use, AVN-944 (26), paradoxically exerted preferential inhibition of $\mathrm{Tsc}^{+/+}$cell growth (Supplemental Figure $1 \mathrm{H}$ ). Mizoribine also exhibited greater selectivity than MPA in 3 isogenic pairs of TSC2-deficient or -expressing cell lines: a murine Tsc2 $^{-/-}$renal tumor-derived cell line (105K cell line) and a human $\mathrm{TSC}^{-1-}$ renal angiomyolipoma-derived cell line (621101 cell line), both stably reconstituted with either wild-type TSC2 or empty vector, and HeLa cells with stable shRNA-mediated knockdown of TSC2 or nontargeting control (Figure 1B and Supplemental Figure 1, I and J). Importantly, these effects on viable cell number reflect selective induction of apoptosis by mizoribine in $T s c 2^{-1-}$ cells, as measured by caspase-3 cleavage and annexin V/propidium iodide staining (Figure 1, C and D; and Supplemental Figure 2A). Consistent with previous reports (27, 28), higher doses of MPA and AVN-944 reduced mTORC1 signaling in wild-type cells, as measured by phosphorylation of the mTORC1 substrate S6K, likely due to their reported effects on the protein levels of Rheb ${ }^{36}$, whereas mizoribine did not affect Rheb levels or mTORC1 activity (Figure 1C).

Consistent with our previous findings that IMPDH inhibition caused replication stress and DNA damage preceding the induction of apoptosis specifically in cells with elevated mTORC1 signaling (Supplemental Figure 2B) (18), mizoribine treatment increased the accumulation of $T s c 2^{-/-}$cells in S-phase, with little to no effect on $T s c 2^{+/+}$cells (Supplemental Figure 2, C and D). MPA treatment showed a similar effect but also increased the percentage of $T s c 2^{+/+}$cells in S-phase. Unlike the highly selective effects of mizoribine on $T s c 2^{-/}$cells, MPA also induced low levels of DNA damage and apoptosis in $T s c 2^{+/+}$cells, as indicated by phosphorylation of histone H2AX and cleavage of caspase-3, respectively (Supplemental Figure 2E). Correlating with the induction of apoptosis, mizoribine and MPA treatment increased proapoptotic Bc12 family proteins, especially BIM, and modestly decreased antiapoptotic proteins in $T s c 2^{-/}$cells, with MPA also having some effect on $T s c 2^{+/+}$cells (Supplemental Figure 2E). Consistent with previous studies (7), c-Myc levels were substantially lower in $T s c 2^{-1-}$ cells, even in vehicle-treated cells.

Differences in selectivity among the clinically used IMPDH inhibitors in these cell culture studies could not be explained by varying effects on purine nucleotide levels, as mizoribine, MPA, and ribavirin caused similar reduction in guanylates, with only minor effect on adenylates, at doses that yield comparable effects on $\mathrm{Tsc}^{-/-}$cell proliferation (Figure 2A). AVN-944 reduced guanylates but also more strongly reduced adenylates than the other IMPDH inhibitors (Figure 2B). All 4 IMPDH inhibitors caused accumulation of aminoimidazole carboxamide ribonucleotide (AICAR), an intermediate in the de novo purine synthesis pathway upstream of IMPDH (Figure 2C and Supplemental Figure 2F). Consistent with active mTORC1 increasing metabolic flux through the de novo purine synthesis pathway (4), AICAR accumulated to a greater extent in $T s c 2^{-/}$cells compared with $T s c 2^{+/+}$cells with all 4 treatments. Importantly, the effects of both mizoribine and MPA on DNA damage and apoptosis were blocked by cotreatment with rapamycin, demonstrating that the sensitivity of $T s c 2^{-/}$cells to these inhibitors is dependent on sustained mTORC1 activity (Supplemental Figure 2G). The effects of mizoribine and MPA on cell proliferation, DNA damage, and apoptosis were all rescued by addition of excess exogenous guanosine, which is converted within cells to GMP, independent of IMPDH, confirming that their effects stem from guanylate nucleotide depletion (Figure 2D and Supplemental Figure 2H).

Mizoribine is superior to mycophenolate in mouse TSC tumor models. Given that mizoribine and MPA were the 2 most selective nucleotide synthesis inhibitors for targeting TSC2-deficient cells compared with wildtype cells in culture and that they are used clinically for similar indications $(29,30)$, differing from ribavirin (which has other known mechanisms of action) $(24,25)$, we directly compared their effects in vivo using 


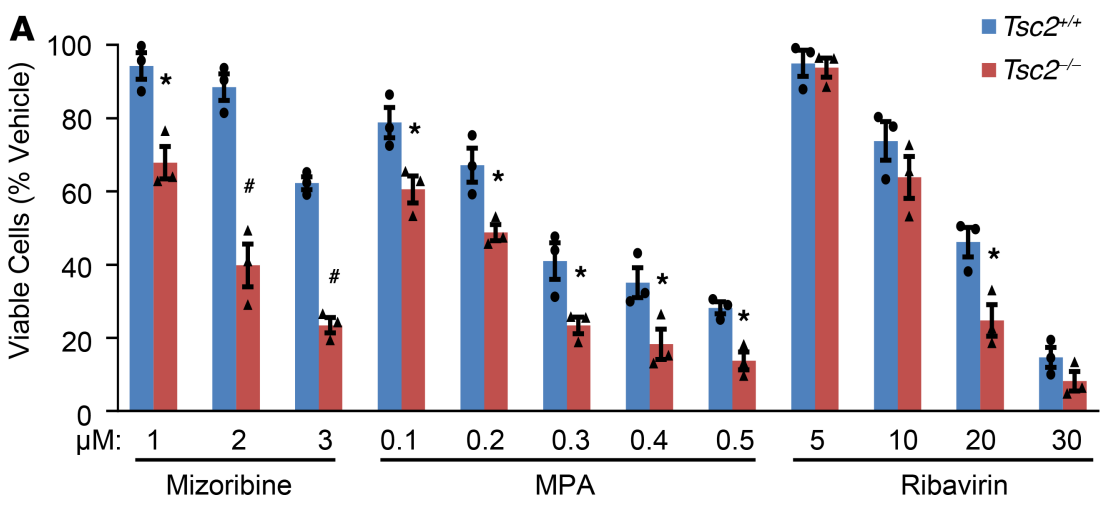

C

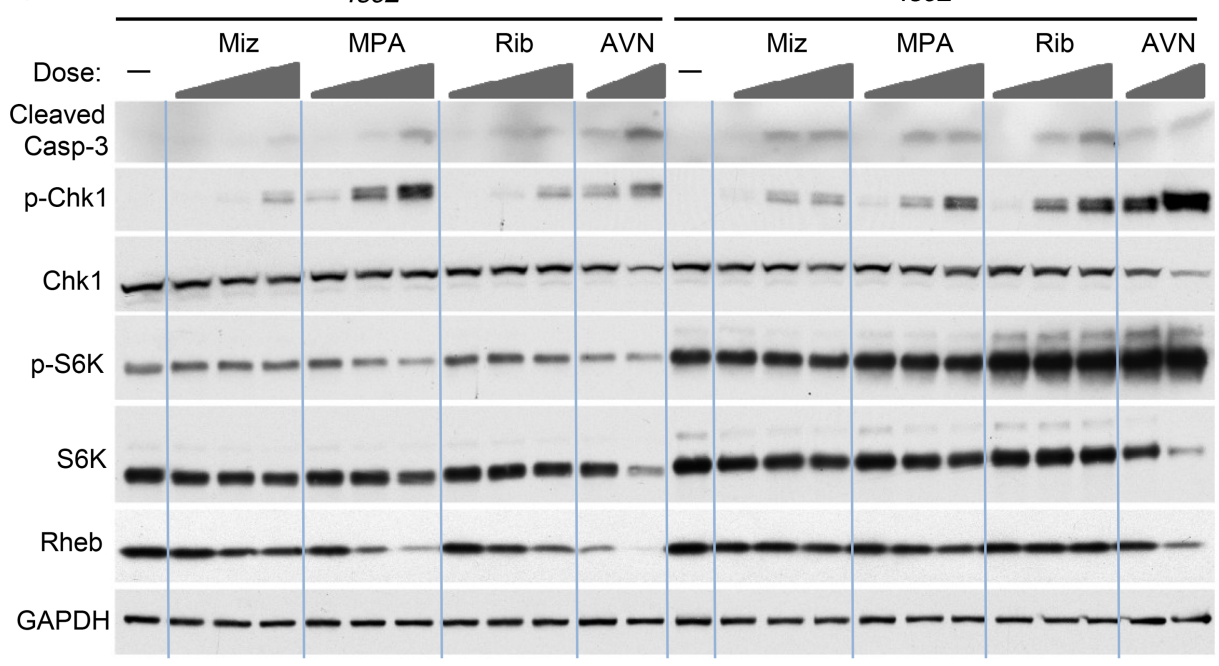

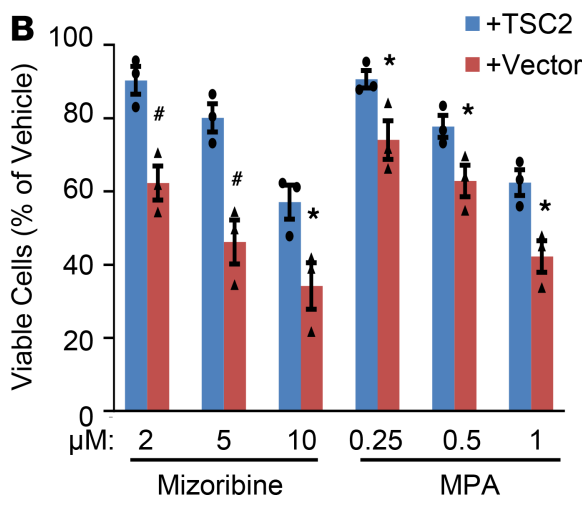

D

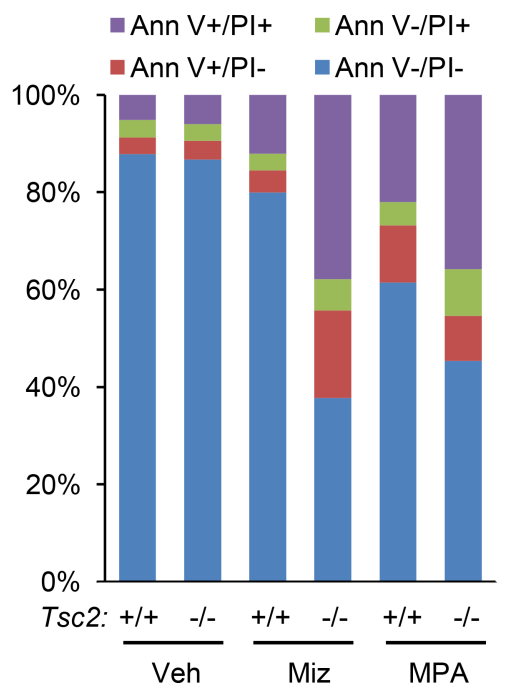

Figure 1. Mizoribine is the most selective IMPDH inhibitor for reducing the viability of TSC2-deficient cells in culture. (A) Littermate-derived Tsc2 ${ }^{+/+}$ and $\mathrm{TsC2}^{-1-}$ mouse embryonic fibroblasts (MEFs) or (B) Tsc2 ${ }^{-1-} 105 \mathrm{~K}$ renal tumor-derived cells stably reconstituted with empty vector or wild-type TSC2 were treated with vehicle or given concentrations of the indicated IMPDH inhibitors for (A) 72 hours or (B) 48 hours. Viable cells were counted by trypan blue exclusion and graphed as percentage of vehicle-treated cells. $n=3$ independent experiments. (C) Cells in $\mathbf{A}$ were treated for 24 hours with vehicle, mizoribine (Miz: 1, 2, or $3 \mu \mathrm{M}$ ), mycophenolic acid (MPA: 125, 250, or $500 \mathrm{nM}$ ), ribavirin (Rib: 10, 20, or $30 \mu \mathrm{M}$ ), or AVN-944 (AVN: 100 or $250 \mathrm{nM}$ ) followed by immunoblotting for indicated proteins. Results are representative of at least 2 independent experiments. S6K, S6 kinase. (D) Annexin V (Ann V)/propidium iodide (PI) staining on cells in A treated for 72 hours with vehicle, $3 \mu \mathrm{M}$ mizoribine, or $250 \mathrm{nM}$ MPA. $n=3$ independent experiments. Graphical data are presented as mean of indicated replicates \pm SEM. ${ }^{*} P<0.05$, ${ }^{*} P<0.01$ by 2 -tailed Student's $t$ test.

distinct TSC2-deficient models of mTORC1-driven tumor growth. Mice bearing xenograft tumors established with the $T s 2^{--}$renal tumor-derived $105 \mathrm{~K}$ cell line were treated daily with vehicle, mizoribine (75 $\mathrm{mg} / \mathrm{kg}$ by i.p. injection), or the FDA-approved MPA prodrug MMF (100 mg/kg by oral gavage) for 20 days. These treatments were also compared with the mTORC1 inhibitor rapamycin $(1 \mathrm{mg} / \mathrm{kg}$ Monday, Wednesday, and Friday [MWF] by i.p. injection), which has been shown previously to be effective in this tumor model and is used to treat tumors in patients with TSC (31). Treatment was initiated when tumors first became palpable and measurable (Figure $3 \mathrm{~A}$ ). Tumor growth was completely halted by mizoribine or rapamycin treatment, while only modestly slowed by MMF (Figure 3B). Total body weight was not affected in any treatment group (Supplemental Figure 3A). Plasma concentrations of mizoribine and MPA (the active product of MMF) were measured at the end of the treatment phase by LC-MS/MS and quantified using standard curves (Supplemental Figure 3, B and C). Plasma concentrations of both compounds were well within the therapeutically achievable ranges typically used for immunosuppression in humans (Figure 3C) (32-35). Tumor immunohistochemical (IHC) staining revealed a slight reduction in the proliferation marker Ki-67 in MMF-treated tumors compared with vehicle but no increase in tumor cell death as determined by $\mathrm{H} \& \mathrm{E}$ and cleaved caspase-3 staining (Figure 3D and Supplemental Figure 3, D and E). Similar to cells in culture (Figure 1C), MMF slightly reduced phosphorylation of the direct mTORC1 substrate S6K and the 
A

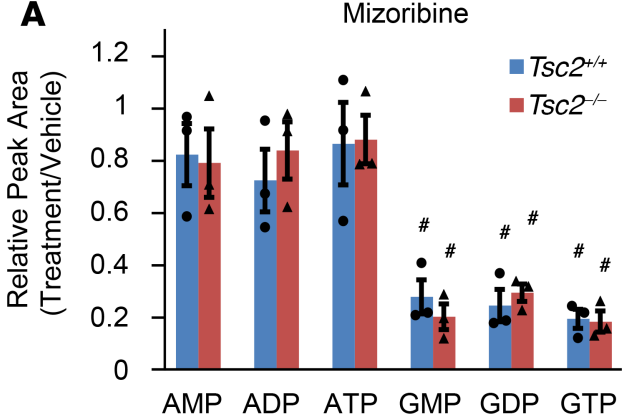

B
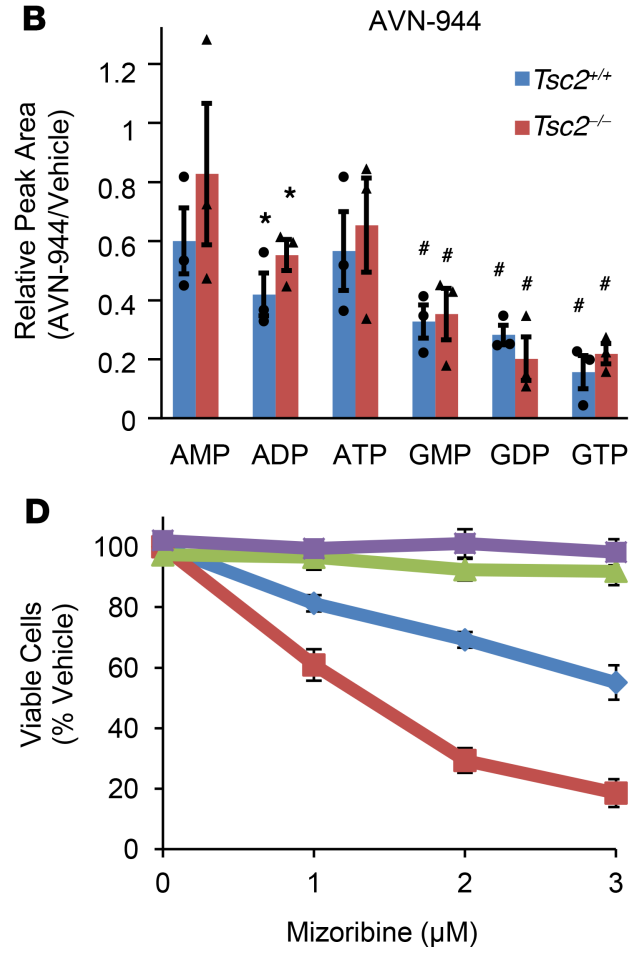

MPA
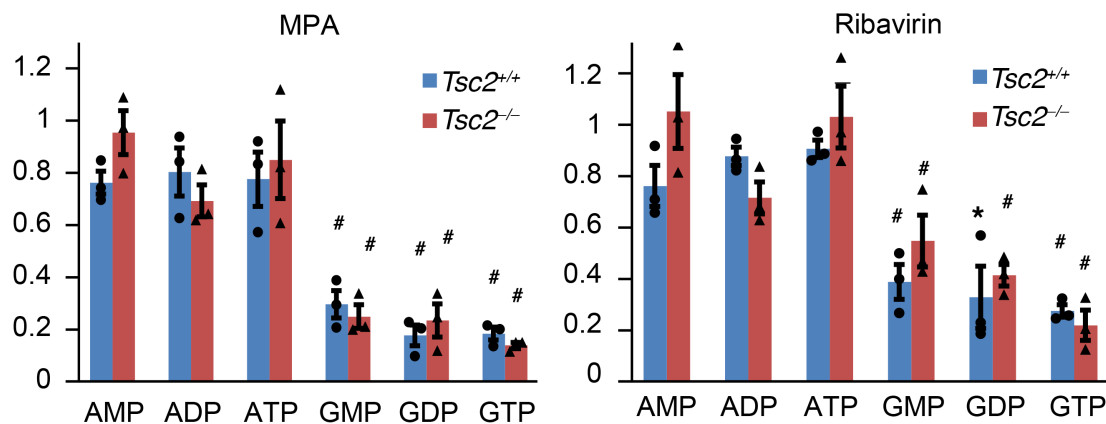

Figure 2. Distinct IMPDH inhibitors have similar effects on purine metabolism in vitro. (A and B) Guanylate and adenylate nucleotides in littermate-derived $\mathrm{TSC2}^{+/+}$and Tsc2-/- MEFs measured by liquid chromatography tandem mass spectrometry (LC-MS/MS) following 12-hour treatment, where indicated, with vehicle, mizoribine $(2 \mu \mathrm{M})$, MPA (250 nM), ribavirin $(20 \mu \mathrm{M})$, or AVN-944 (250 nM) and graphed relative to vehicle. $n=3$ biological replicates from a single experiment. (C) AICAR levels relative to vehicle-treated cells in samples from $\mathbf{A}$ and $\mathbf{B}$. (D) Viable cell counts in cells treated for 72 hours with indicated concentrations of mizoribine or MPA with or without addition of exogenous guanosine (guan, $50 \mu \mathrm{M}$ ). $n=3$ independent experiments. Graphical data are presented as mean of indicated replicates \pm SEM. ${ }^{*} P<0.05,{ }^{*} P<0.01$ by 2 -tailed Student's $t$ test.

S6K substrate ribosomal protein S6 (Figure 3, D and E). Because rapamycin-treated tumors are known to resume growth when treatment is discontinued, we compared the rate of tumor regrowth in the mizoribine and rapamycin groups to assess the durability of the antitumor response to these compounds. Rather than resecting tumors after the final treatment, tumors in these groups were allowed to regrow for up to 38 days, until they neared maximum allowable size (Figure 3A). Importantly, mizoribine-treated tumors regrew more slowly than those treated with rapamycin (Figure 3B). At the end of the regrowth phase, despite not being treated for several weeks, tumors in the mizoribine group contained large necrotic regions, which were not observed in those initially treated with rapamycin (Figure 3F). These data indicate that mizoribine exerts a more durable cytotoxic effect on the tumor cells in this model, as compared with the established cytostatic effects of rapamycin $(8,13,31)$.

Because rapamycin and its analogs are frequently prescribed to treat tumors in patients with TSC, we asked whether previous rapamycin treatment would affect the response of TSC2-deficient tumors to mizoribine and MMF. Leveraging a second widely used TSC tumor model that exhibits rapid tumor regrowth upon halting rapamycin treatment, xenograft tumors were established using the TSC2-deficient rat uterine 
A

\begin{tabular}{l|c|c|}
\multicolumn{3}{c}{ NSG Mice } \\
\begin{tabular}{|c|c|} 
Tumor \\
Development
\end{tabular} & Treat & $\begin{array}{c}\text { Tumor } \\
\text { regrowth }\end{array}$ \\
\hline 1 month & 20 days & 38 days \\
Inject & Established Stop \\
$105 \mathrm{~K}$ & tumors treatment \\
cells & &
\end{tabular}

B

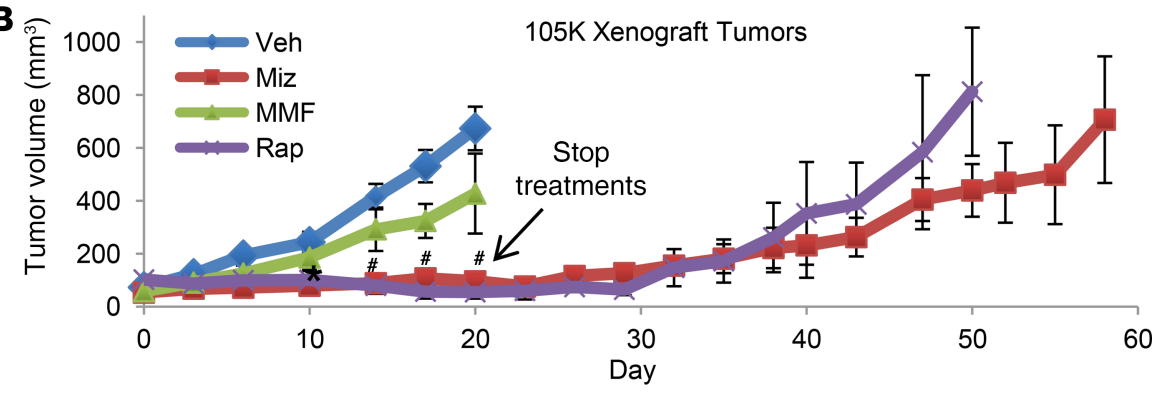

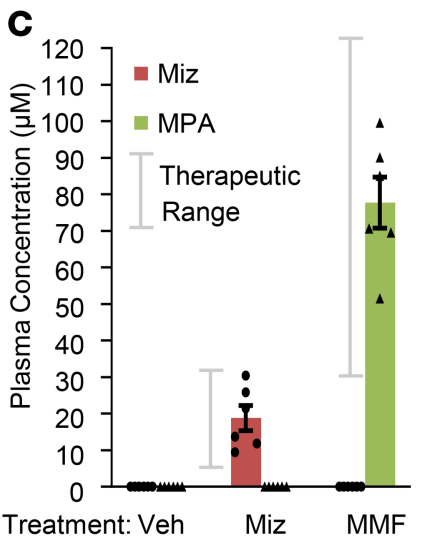

E

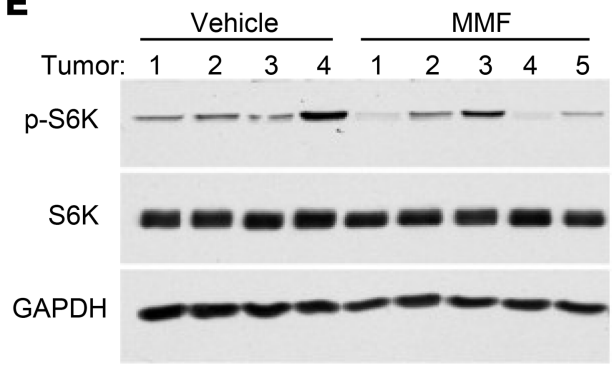

D
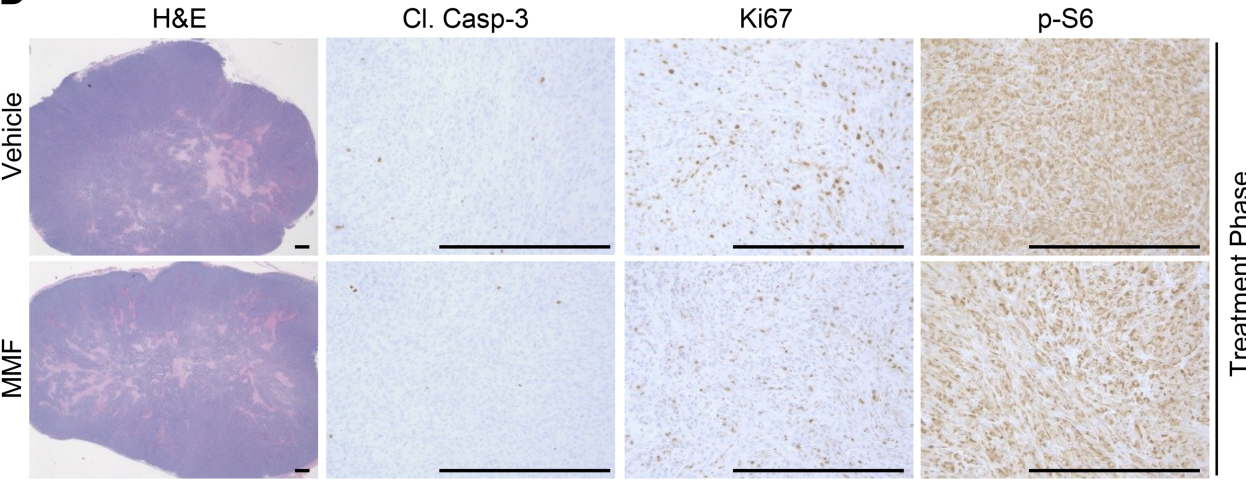

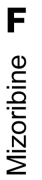
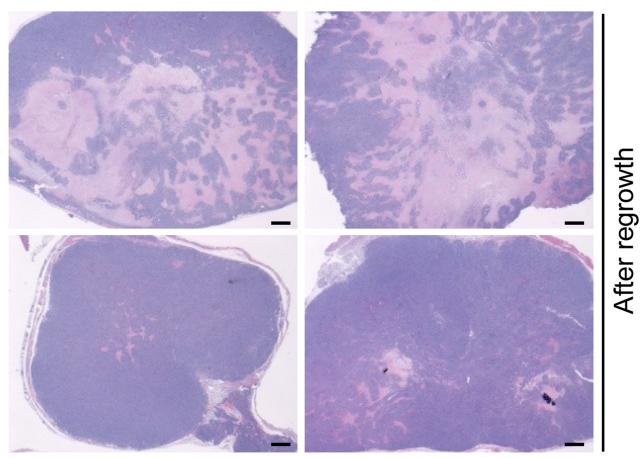

Figure 3. Mizoribine is superior to mycophenolate in TSC2-deficient 105K xenograft tumors at therapeutically relevant concentrations. (A) Experimental design used in B-F. NOD.Cg-Prkd $c^{\text {scid }} / 12 \mathrm{rg}^{\text {tmiWil }} / \mathrm{Sz}$ (NSG) mice bearing 105K cell xenograft tumors were treated for 20 days with vehicle, mizoribine (75 mg/ $\mathrm{kg} / \mathrm{d}$ by i.p. injection), MMF (100 mg/kg/d by oral gavage), or rapamycin (1 mg/kg MWF by i.p. injection). Treatments were discontinued after 20 days, and tumors in the mizoribine and rapamycin groups were allowed to regrow. (B) Tumor volume measured every third day. $n=6$ mice per group. (C) Mizoribine and MPA concentrations in blood plasma collected 2.5 hours after the final treatment, measured by LC-MS/MS. $n=6$ mice per group. (D) Representative H\&E and IHC staining on tumors from vehicle- or MMF-treated mice resected 3 hours after the final treatment. (E) Immunoblotting of tumor extracts from vehicle- or MMF-treated mice resected 3 hours after final treatment. (F) Representative H\&E staining of tumors from mizoribine- or rapamycin-treated mice resected after regrowth. Graphical data are presented as mean of indicated replicates \pm SEM. ${ }^{*} P<0.05,{ }^{\#} P<0.01$ by 2 -tailed Student's $t$ test. Scale bars: $0.5 \mathrm{~mm}$.

leiomyoma-derived ELT3 cell line. Tumor-bearing mice were treated as above for 26 days with vehicle, mizoribine, or MMF or 23 days with rapamycin. On day 24, the rapamycin-treated mice were switched to vehicle, mizoribine, or MMF treatment for another 17 days (Figure 4A). Consistent with the findings above (Figure 3B), mizoribine had a greater effect on ELT3 xenograft tumor growth than MMF, and prior rapamycin treatment did not influence this response (Figure 4, B and C). Again, these treatments did not affect total body weight (Supplemental Figure 3F), and final plasma mizoribine and MPA concentrations were similar to those measured in the $105 \mathrm{~K}$ tumor-bearing mice (Figure 4D). These results indicate that the antitumor activity of IMPDH inhibitors, and superiority of mizoribine over MMF, are not affected by previous rapamycin treatment of TSC tumors.

To compare the accessibility of mizoribine and MPA to the tumors in this model, we determined the levels of these compounds in tumor metabolite extracts relative to those measured in plasma. The tumor-to-plasma ratio of mizoribine was nearly identical to that of MPA in mice treated with each respective drug (Figure 4E), while the liver-to-plasma ratio was 4-fold higher for mizoribine than MPA 
A

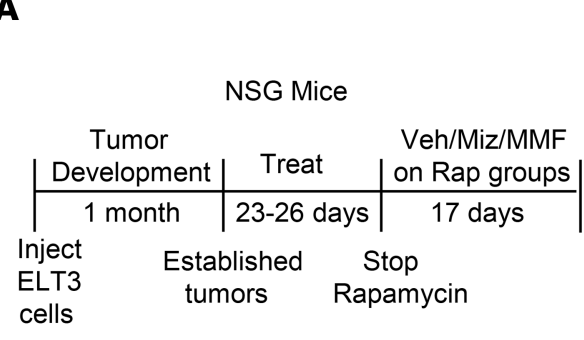

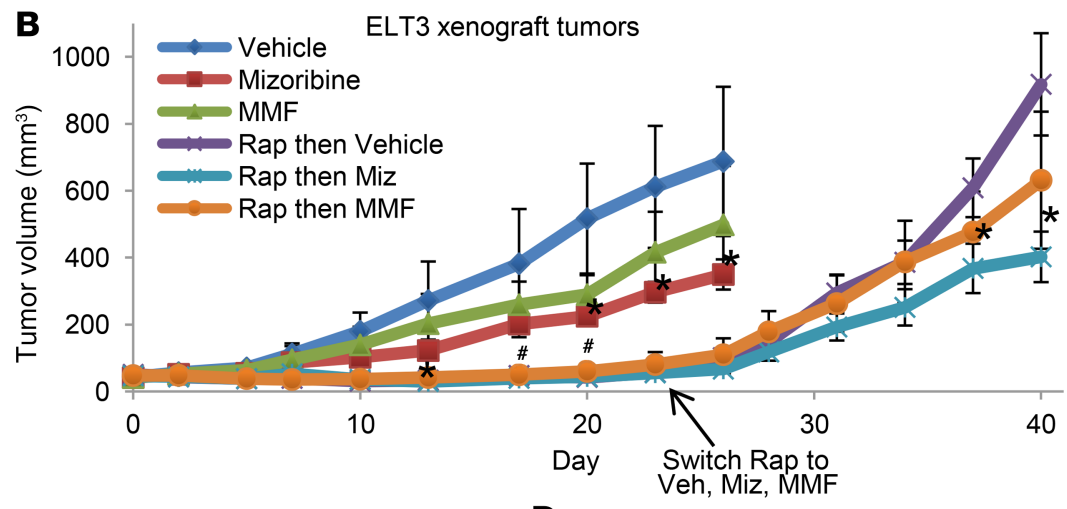

C

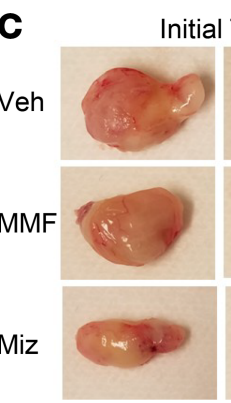

E

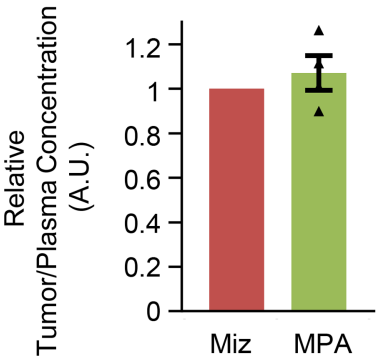

B

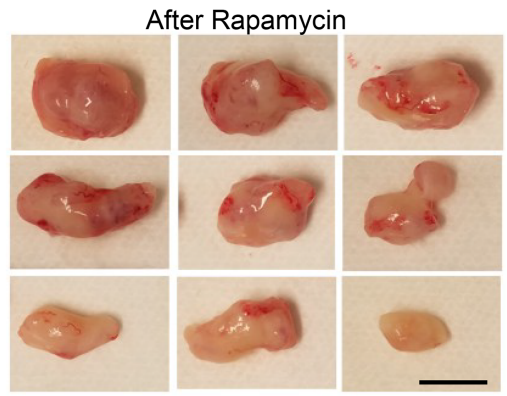

$\mathbf{F}$
D

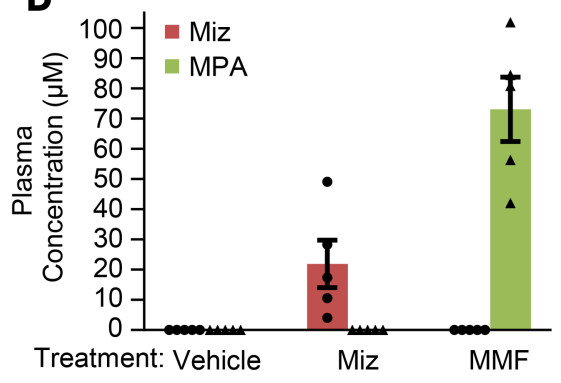

Figure 4. Mizoribine is superior to mycophenolate in TSC2-deficient ELT3 xenograft tumors with or without prior treatment with rapamycin. (A) Experimental design used in B-F. NSG mice bearing ELT3 cell xenograft tumors were treated for 26 days with vehicle, mizoribine (75 mg/kg/d by i.p. injection), or MMF (100 $\mathrm{mg} / \mathrm{kg} / \mathrm{d}$ by oral gavage) or for 23 days with rapamycin ( $1 \mathrm{mg} / \mathrm{kg}$ MWF by i.p. injection). On day 23, rapamycin treatment was discontinued, and those mice were switched to vehicle, mizoribine, or MMF treatment, as above, for 17 days. (B) Tumor volume measured every third day. $n=6$ mice per group. (C) Images of representative tumors resected at the end of the indicated treatments. Scale bars: $1 \mathrm{~cm}$. (D) Mizoribine and MPA concentrations in blood plasma collected on day 26 , 2.5 hours after the final treatment and measured by LC-MS/MS. $n=5$ mice per group. (E and $\mathbf{F}$ ) Peak area values of mizoribine and MPA in tumor (E) and liver (F) metabolite extracts were normalized to those in plasma from the same mice. The mean tumor/plasma (E) or liver/plasma (F) ratio of MPA is shown relative to mizoribine. $n=4$ mice per group. Graphical data are presented as mean of indicated replicates \pm SEM. ${ }^{*} P<0.05$, ${ }^{*} P<0.01$ by 2 -tailed Student's $t$ test.

in the same mice (Figure 4F). Thus, the reduced antitumor efficacy of MMF relative to mizoribine is not due to reduced MPA availability to tumors.

Mizoribine and mycophenolate have differential effects on tumor metabolism. To gain insight into the underlying cause of the differential antitumor response between mizoribine and MMF in these TSC tumor models, we profiled steady-state metabolite levels in the ELT3 xenograft tumors after 26 days of treatment using LC-MS/MS-based metabolomics (tumor metabolites significantly changed relative to vehicle are shown in Figure 5, A and B). Of the tumor metabolites significantly changed in response to mizoribine and MMF, $51 \%$ and $41 \%$, respectively, were shared between the 2 treatments (18 total, indicated by asterisk, Figure 5, A and B). For instance, both mizoribine and MMF increased tumor putrescine and $N$-acetyl spermidine levels, consistent with a recent report demonstrating that the IMPDH inhibitor ribavirin can stimulate an increase in these polyamines (36). While both drugs decreased tumor GMP levels, mizoribine had markedly stronger effects on tumor GDP and GTP than MMF, with the tumors from MMF-treated mice exhibiting modestly lower levels of adenylates relative to vehicle or mizoribine (Figure $5 \mathrm{C}$ ). With a 20 -fold increase, AICAR was the most significantly elevated metabolite $(P<0.0003)$ in mizoribine-treated tumors, and consistent with our previous findings, mizoribine treatment also increased plasma AICAR 

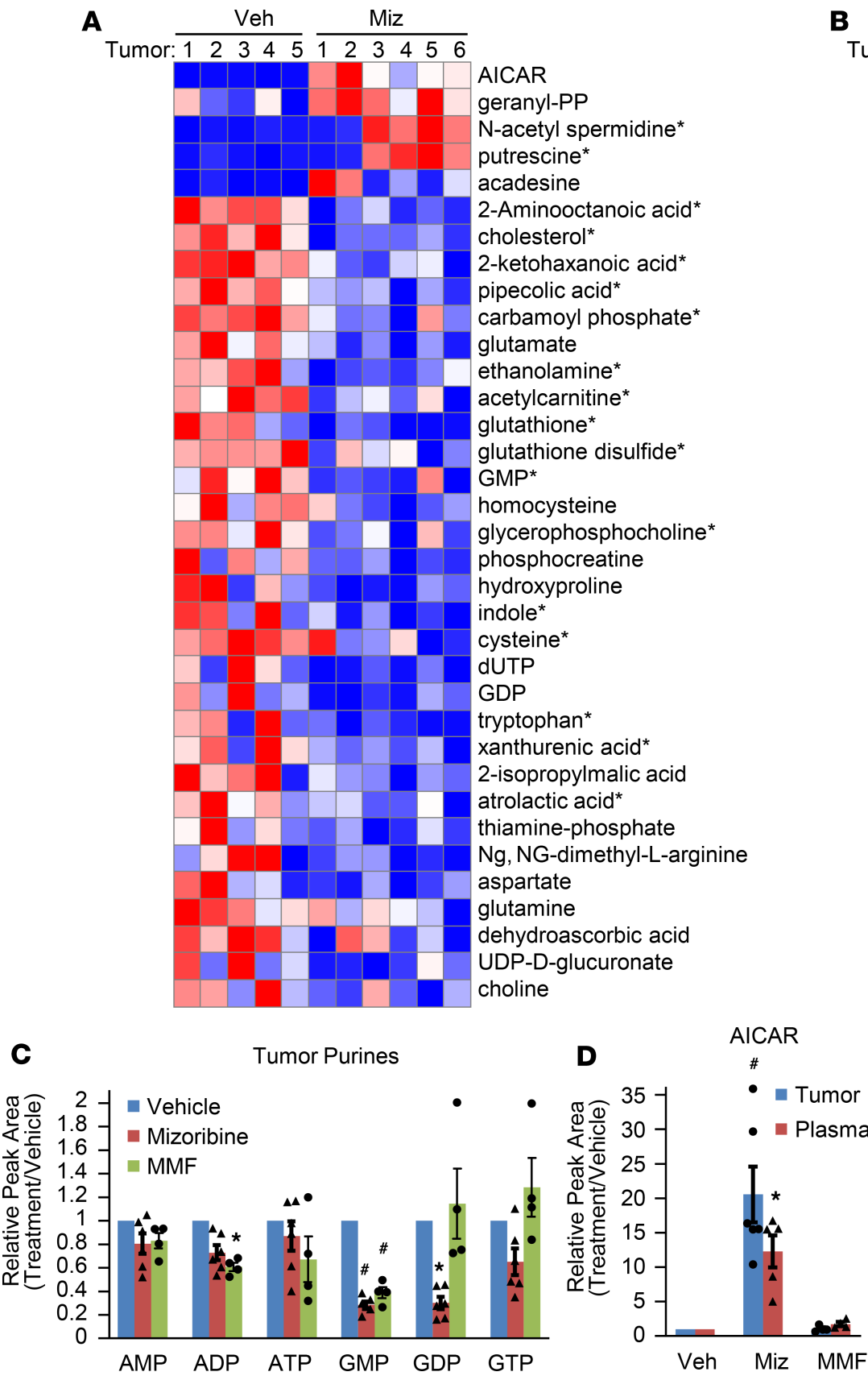

B Tumor: $\frac{\text { Veh }}{123345} \frac{\text { MMF }}{12234}$

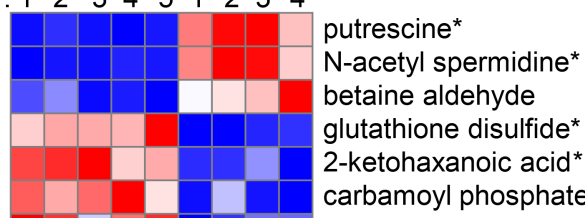

carbamoyl phosphate*

carnitine

adenylosuccinate

cholesterol* $^{*}$

2-Aminooctanoic acid ${ }^{*}$

acetylcarnitine*

glutathione*

GMP*

guanidoacetic acid

CMP

glycerophosphocholine*

NADP+

atrolactic acid*

orotate

spermidine

PRPP

1-methyl-histidine

cellobiose

xanthurenic acid *

kynurenine

creatine

phenylpropiolic acid

taurine

ethanolamine*

maleic acid

taurodeoxycholic acid

phenylalanine

indole*

O8P-01P

alanine

malate

ADP

pipecolic acid*

dCTP

thymine

trehalose-sucrose

tryptophan*

UDP-D-glucose

cysteine*

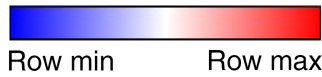

Row min

Figure 5. Effects of mizoribine and MMF on tumor metabolites. (A and B) Steady-state LC-MS/MS-based metabolite profiling of ELT3 xenograft tumors collected from the mice in Figure 4B on day 26, 3 hours after final treatment with vehicle $(n=5)$, mizoribine $(n=6)$, or MMF $(n=4)$. Row normalized heat maps are shown for all significantly changed metabolites $(P<0.05)$ in tumors from $(\mathbf{A})$ mizoribine- or $(\mathbf{B})$ MMF-treated mice relative to vehicle. Metabolites are grouped as increasing or decreasing with treatment and then listed from lowest to highest $P$ value. ${ }^{*}$ Metabolites changed with both mizoribine and MMF. (C and $\mathbf{D})$ Peak area values relative to vehicle of (C) adenylate and guanylate nucleotides in tumors and (D) AICAR in tumors and plasma from the treated mice in $\mathbf{A}$ and $\mathbf{B}$. Graphical data are presented as mean of indicated replicates \pm SEM. ${ }^{*} P<0.05,{ }^{\#} P<0.005$ by 2 -tailed Student's $t$ test.

levels (Figure 5D). However, in contrast to MPA-treated cells in culture (Figure 2C), MMF did not cause significant AICAR accumulation in tumors or plasma (Figure 5, B and D).

In the $105 \mathrm{~K}$ tumor model, 20 days of MMF treatment failed to reduce tumor GMP levels but resulted in significant depletion of tumor GDP and GTP, as well as ADP and ATP (Supplemental Figure 4A). In these tumors, MMF caused only minor (1.6-fold) accumulation of AICAR (Supplemental Figure 4B). As in the ELT3 tumor-bearing mice, mizoribine, but not MMF, caused accumulation of plasma AICAR (Supplemental Figure 4C). Taken together, the decrease in tumor guanylates and increase in polyamines suggest 
that MMF treatment, like mizoribine, is directly affecting IMPDH in these 2 TSC tumor models. However, the greater depletion of tumor adenylates, without effects on AICAR, with MMF treatment indicates distinct metabolic responses that might contribute to the differential antitumor responses measured between these 2 clinically used IMPDH inhibitors. To determine whether these differential metabolic effects might reflect distinct properties of MMF compared with MPA, we treated Tsc2 ${ }^{-1-}$ MEFs with equimolar doses of MMF or MPA, which yielded similar intracellular MPA levels (Supplemental Figure 4D), and compared them with mizoribine treatment. Like mizoribine, both MMF and MPA caused specific depletion of guanylate nucleotides in cultured cells, with only minor effects on adenylates, and caused accumulation of AICAR (Supplemental Figure 4, E and F). Thus, relative to cell culture models and mice treated with mizoribine, the limited depletion of tumor guanylates in mice treated with MMF, and depletion of adenylates and lack of AICAR accumulation, are metabolic effects specific to in vivo administration of MMF.

To more closely compare the plasma and tissue bioavailability of mizoribine and MMF, nontumor-bearing mice were analyzed after 7 days of treatment. Plasma concentrations of mizoribine and MPA were similar to those observed in our previous experiments with prolonged (20- or 26-day) treatments (Figure 3C, Figure 4D, and Supplemental Figure 4G). Mizoribine, but not MMF, treatment caused AICAR accumulation to varying degrees in all tissues examined (plasma, liver, spleen, kidney, brain, lung, and heart), with the greatest accumulation observed in the liver, which is believed to be a major site of de novo nucleotide synthesis (Supplemental Figure $4 \mathrm{H})(37,38)$. It is interesting to note that the spleen is the only tissue where MMF treatment increased AICAR levels, although much less so than mizoribine, perhaps reflecting more efficient targeting of this key organ of the immune system. Pharmacokinetic analysis after a single treatment with mizoribine or MMF revealed that despite a higher peak plasma concentration of MPA and slower clearance compared with mizoribine, plasma AICAR only accumulated after mizoribine treatment and did not return to baseline levels even after 24 or 48 hours (Supplemental Figure 4, I-L). These data suggest that AICAR accumulation is a rapidly induced, long-lived biomarker of IMPDH inhibition by mizoribine in plasma, normal tissues, and tumors.

Mizoribine is superior to the maximum tolerated dose of MMF in an immunocompetent syngeneic TSC tumor model. Given the established immunosuppressive effects of IMPDH inhibitors, we compared mizoribine to MMF in an immunocompetent syngeneic xenograft tumor model and also asked whether higher doses of MMF might have efficacy similar to mizoribine. We also controlled for the route of drug administration by delivering both mizoribine and MMF orally, recapitulating their most common delivery method in patients. We found that plasma mizoribine concentration and AICAR levels were higher when mizoribine was administered by oral gavage compared with the same dose administered via i.p. injection (Supplemental Figure 5, A and B); thus the dose was reduced for oral delivery to yield plasma concentrations similar to those obtained with i.p. injection (Figure 6A). In a dosing pilot experiment, wild-type C57BL/6J mice were treated by oral gavage with vehicle, $50 \mathrm{mg} / \mathrm{kg} / \mathrm{d}$ mizoribine, $100 \mathrm{mg} / \mathrm{kg} / \mathrm{d}$ MMF, $300 \mathrm{mg} / \mathrm{kg} / \mathrm{d} \mathrm{MMF}, 500 \mathrm{mg} / \mathrm{kg} / \mathrm{d} \mathrm{MMF}$, or $1 \mathrm{mg} / \mathrm{kg}$ Q2D rapamycin for 10 days. Although plasma MPA concentrations increased in a dose-dependent manner, the 5-fold increase in MMF dose yielded a less than 2-fold increase in plasma MPA concentration, suggesting a bioavailability limitation (Figure 6A). Consistent with this, at lower doses, doubling the MMF dose from 25 to $50 \mathrm{mg} / \mathrm{kg} / \mathrm{d}$ correspondingly doubled the resulting plasma MPA concentration, but doubling MMF from 50 to $100 \mathrm{mg} / \mathrm{kg} / \mathrm{d}$ increased plasma MPA levels by only 1.4-fold (Supplemental Figure 5C). Importantly, white blood cell counts indicated that the immunosuppressive effects of MMF were intact with this treatment, with the lymphocyte population decreasing in a dose-dependent manner (Figure 6B). The chosen doses of rapamycin and mizoribine yielded the same effects as 100 and $300 \mathrm{mg} / \mathrm{kg} \mathrm{MMF}$, respectively, on total white blood cells. Consistent with our findings in immunocompromised mice, mizoribine robustly increased plasma AICAR levels while MMF had only slight effects (Supplemental Figure 5D).

C57BL/6J mice bearing syngeneic $105 \mathrm{~K}$ xenograft tumors were treated orally for 24 days with vehicle, mizoribine $(50 \mathrm{mg} / \mathrm{kg} / \mathrm{d})$, or $\operatorname{MMF}(100,300$, or $500 \mathrm{mg} / \mathrm{kg} / \mathrm{d})$, compared with rapamycin $(1 \mathrm{mg} / \mathrm{kg}$ MWF, i.p. injection), beginning when tumors first became palpable (Figure 6C). Although MMF treatment slowed tumor growth in a dose-dependent manner, the effect of mizoribine was far superior to even the highest dose of MMF, shrinking tumors by approximately $40 \%$ over the 24-day treatment period (Figure 6D). MMF treatment did not increase tumor apoptosis (Figure 7A and Supplemental Figure 5E), but the modest decrease in tumor growth was accompanied by a dose-dependent decrease in the proliferation marker Ki-67 and the mTORC1 signaling marker phospho-S6 (Figure 7, B and C). Mice treated with the highest dose of MMF (500 mg/kg/d) experienced weight loss until treatment day 10 when every third day 


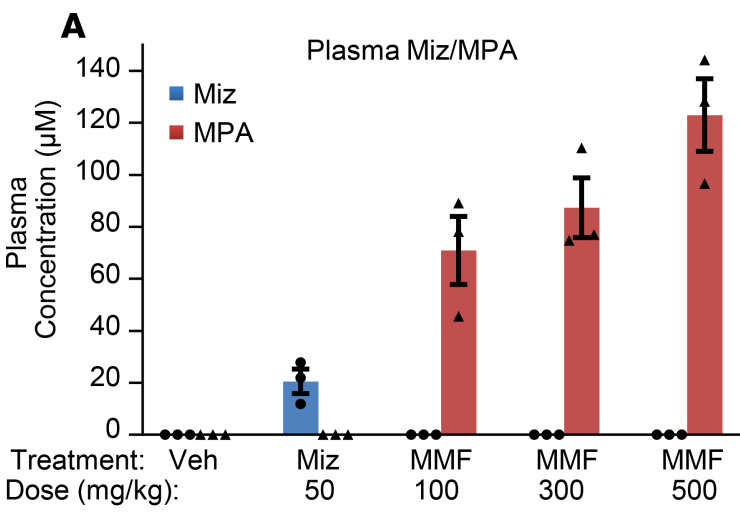

B

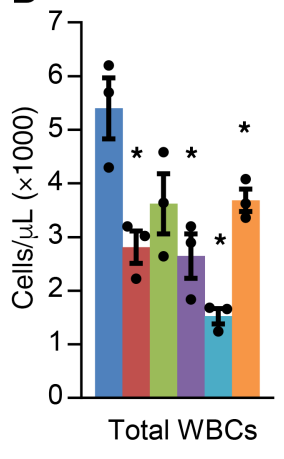

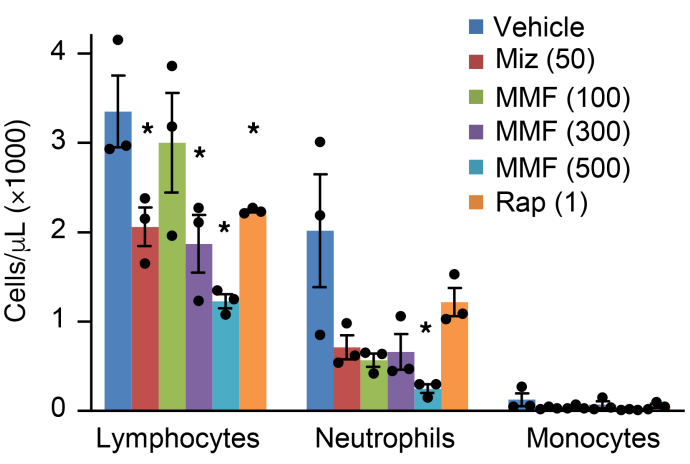

C

\begin{tabular}{|c|c|c|c|}
\hline \multicolumn{4}{|c|}{ C57BL/6J Mice } \\
\hline & $\begin{array}{c}\text { Tumor } \\
\text { Development }\end{array}$ & Treat & $\begin{array}{c}\text { Tumor } \\
\text { regrowth }\end{array}$ \\
\hline & 1 month & 24 days & 41 days \\
\hline $\begin{array}{l}\text { Inject } \\
105 \mathrm{~K} \\
\text { cells }\end{array}$ & $\begin{array}{r}\text { Establ } \\
\text { tumo }\end{array}$ & $\begin{array}{l}\text { lished } s \\
\text { ors tre }\end{array}$ & $\begin{array}{l}\text { Stop } \\
\text { atment }\end{array}$ \\
\hline
\end{tabular}

\section{D}

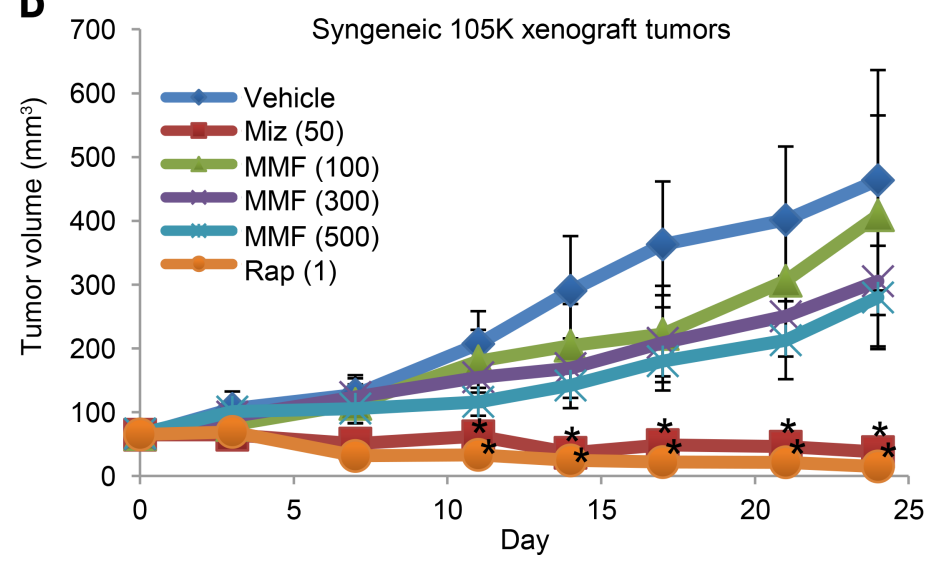

Figure 6. Mizoribine is superior to the maximum tolerated dose of MMF in an immunocompetent syngeneic xenograft model of TSC2-deficient tumor growth. (A and B) C57BL/6) mice were treated for 10 days with mizoribine $(50 \mathrm{mg} / \mathrm{kg} / \mathrm{d}$ by oral gavage) MMF (100, 300, or $500 \mathrm{mg} / \mathrm{kg} / \mathrm{d}$ by oral gavage), or rapamycin ( $1 \mathrm{mg} / \mathrm{kg}$ every 2 days [Q2D] by i.p. injection). Blood and plasma were collected 2.5 hours after the final treatment for measurement of (A) mizoribine and MPA concentrations in plasma by LC-MS/MS and (B) white blood cell counts. $n=3$ mice/group. (C) Experimental design used in $\mathbf{D}$ and in Figure 7. C57BL/ 6 mice bearing syngeneic $105 \mathrm{~K}$ cell xenograft tumors were treated as in $\mathbf{A}$ and $\mathbf{B}$, except rapamycin was administered on MWF, for 24 days, at which point mice were sacrificed or, for the mizoribine and rapamycin groups, treatments were discontinued and tumors were allowed to regrow. $n=6$ mice/group. (D) Tumor volume during the treatment phase measured every third day. Graphical data are presented as mean of indicated replicates \pm SEM. ${ }^{*} P<0.05$ by 2 -tailed Student's $t$ test.

of treatment was skipped in this group to mitigate the toxicity, but weight loss was not observed in any other treatment group (Supplemental Figure 5F). As observed in 105K tumors from immunocompromised mice (Supplemental Figure 4, A and B), MMF reduced tumor GDP, GTP, and ATP levels and did not cause AICAR accumulation (Supplemental Figure 5G).

To directly compare the durability of the response to mizoribine to that of rapamycin, treatment was discontinued for these groups at day 24 and tumors were allowed to regrow (Figure 7D). On the final day of treatment, the mean tumor volume in the mizoribine group was 2.4-fold larger than the rapamycin group. Therefore, although the absolute tumor volume between the mizoribine and rapamycin groups following the regrowth phase was similar (Supplemental Figure $5 \mathrm{H}$ ), tumors from mice previously treated with mizoribine regrew substantially slower than those treated with rapamycin (Figure 7D). Furthermore, consistent with our data from the immunocompromised host (Figure 3F), after treatment withdrawal and tumor regrowth for up to 41 days, the tumors from mice originally treated with mizoribine contained large areas of tumor necrosis and cell death, in stark contrast to the solid tumors arising in mice first treated with rapamycin (Figure 7E).

We previously demonstrated that 1 month of mizoribine treatment reduces the volume and number of renal tumors that develop spontaneously in $T s c 2^{+/-}$mice on the $\mathrm{A} / \mathrm{J}$ strain background (18). To determine whether MMF has antitumor efficacy in this genetic model, $T s c 2^{+/-}$mice were treated for 1 month with MMF (75 mg/kg/d by oral gavage). No effect of MMF treatment on renal tumor volume was observed (Supplemental Figure 5I). We were unable to directly compare MMF to mizoribine in these mice because of $\mathrm{A} / \mathrm{J}$ strain-specific toxicity encountered when mizoribine was administered by oral gavage compared with our previous studies using i.p injection (18), reflecting the observed increase in plasma mizoribine 
A
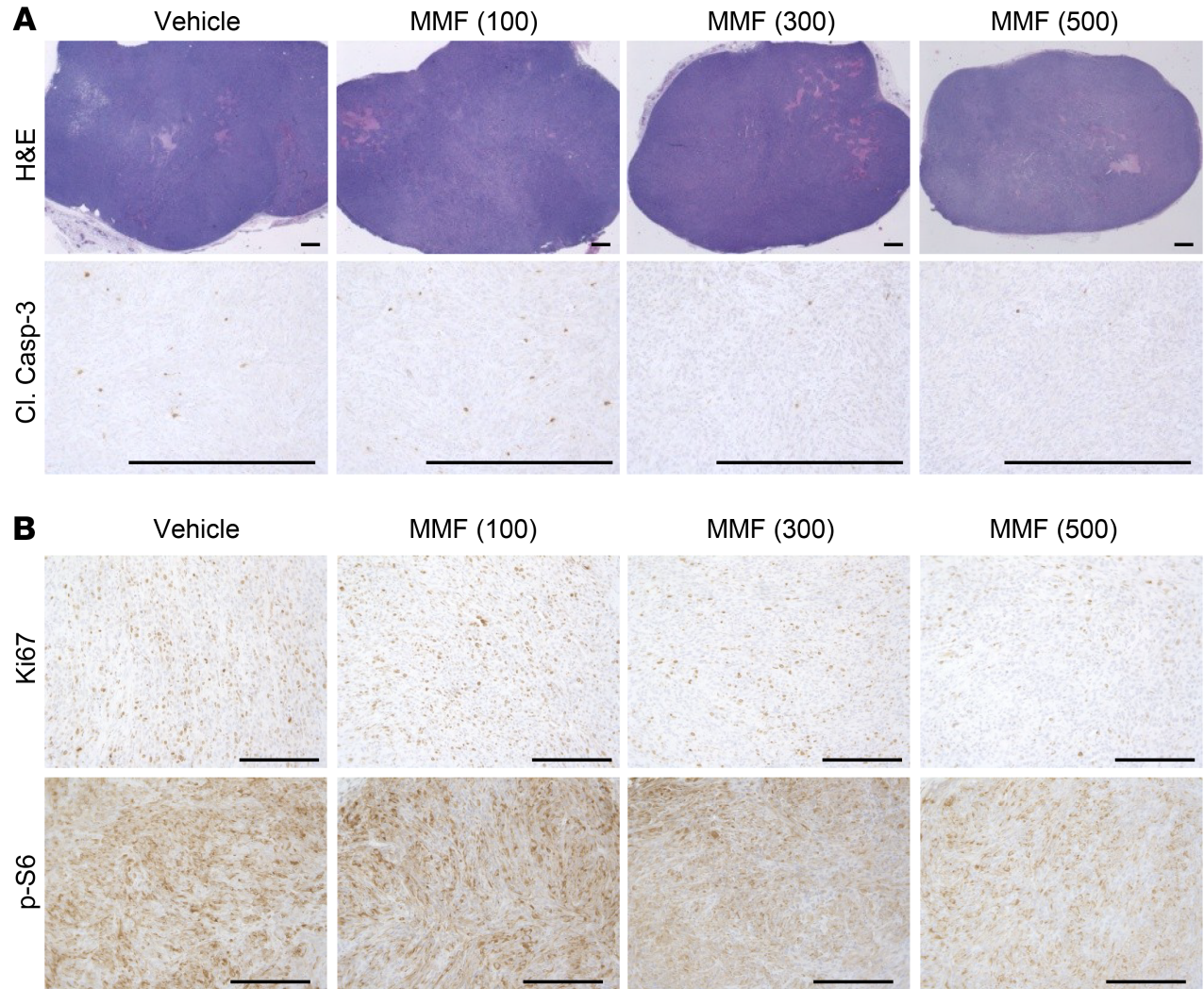

MMF (300)

MMF (500)
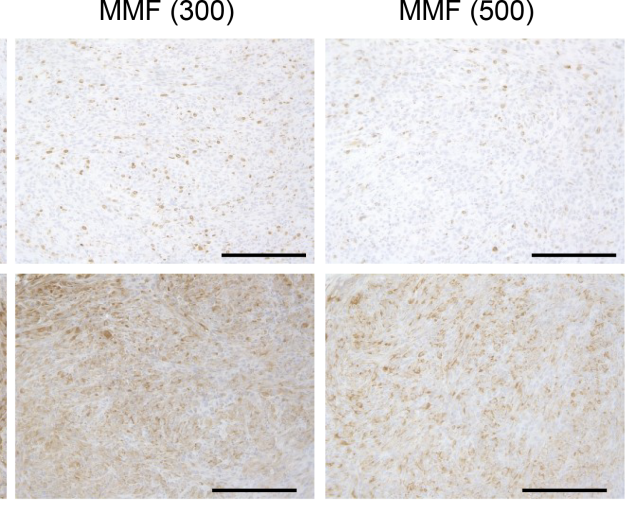

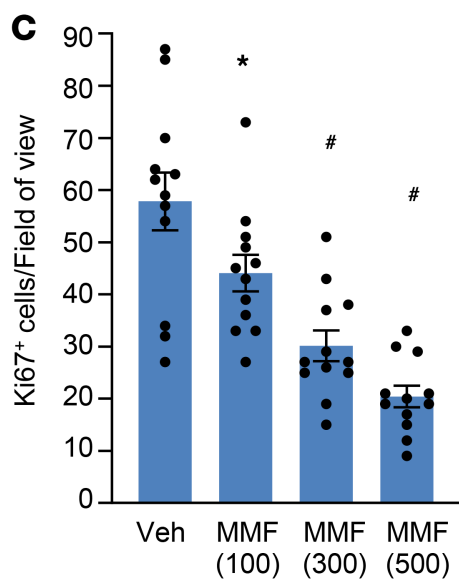

D 60

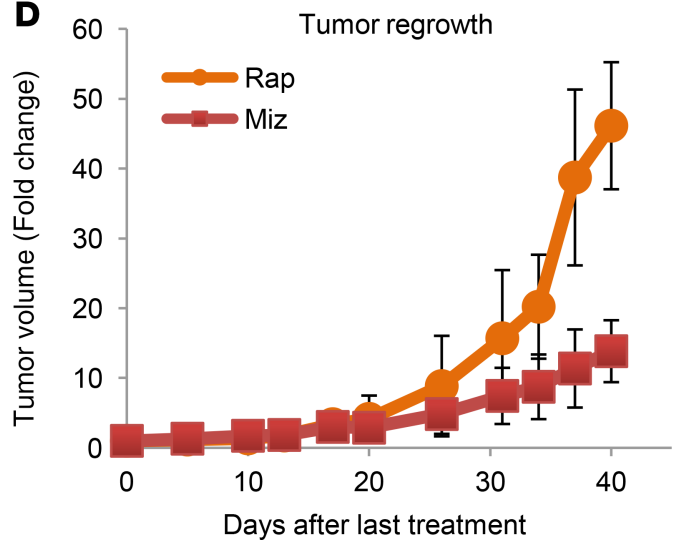

E
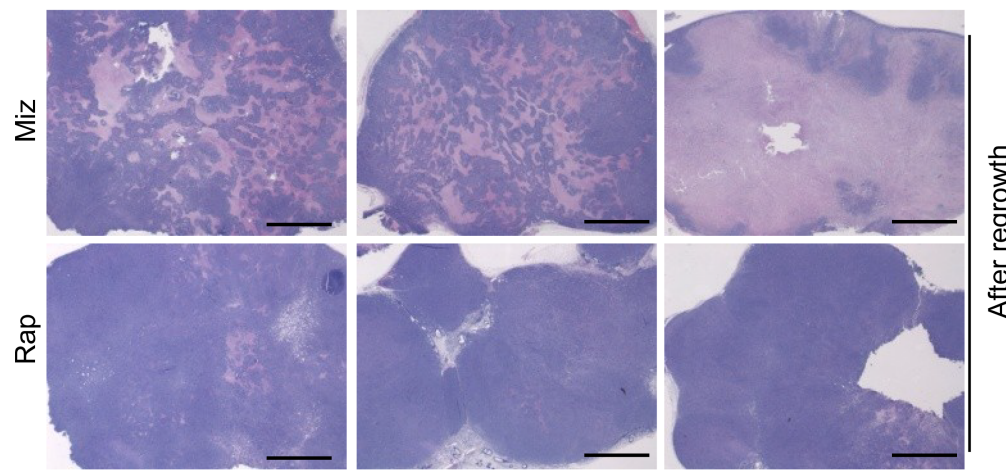

Figure 7. MMF fails to induce cell death and mizoribine treatment elicits a more durable response than rapamycin in a syngeneic TSC tumor model. (A and B) Representative H\&E and IHC staining of tumors from Figure 6D resected 3 hours after the final treatment. Scale bars: $0.5 \mathrm{~mm}$ (A), $0.25 \mathrm{~mm}$ (B). (C) Quantification of Ki-67 staining in B. Staining-positive cells per field of view were counted in 4 tumors per group in a blinded fashion, with 3 nonoverlapping fields counted per tumor. (D) Fold change in tumor volume during the regrowth phase in mice from Figure 6, C and D, relative to the last day of treatment. (E) H\&E staining of tumors from the indicated treatment groups, resected after regrowth (scale bars: 2 mm). Graphical data are presented as mean of indicated replicates \pm SEM. ${ }^{*} P<0.05,{ }^{\#} P<0.01$ by 2 -tailed Student's $t$ test.

concentrations with oral delivery (Supplemental Figure 5, A and B). To determine whether this mizoribine toxicity is specific to $T s c 2^{+/-}$mice, wild-type and $T s c 2^{+/-}$mice on the $\mathrm{A} / \mathrm{J}$ background and wild-type C57BL/6J mice were treated with mizoribine ( $30 \mathrm{mg} / \mathrm{kg} / \mathrm{d}$ by oral gavage) for 1 week. Both the wild-type and $T s c 2^{+/-} \mathrm{A} / \mathrm{J}$ mice experienced similar weight loss, while the total body weight of C57BL/6J mice was unaffected, demonstrating that this effect is strain specific and not related to the $T s c 2^{+/-}$genotype (Supplemental Figure 5J). In this experiment, plasma mizoribine concentration was approximately 4-fold higher in the $\mathrm{A} / \mathrm{J}$ mice, both wild-type and $T s \mathrm{C}^{+/}$, compared with the C57BL/6J mice, thus providing a likely explanation for the increased toxicity in the A/J strain (Supplemental Figure $5 \mathrm{~K}$ ). 
A

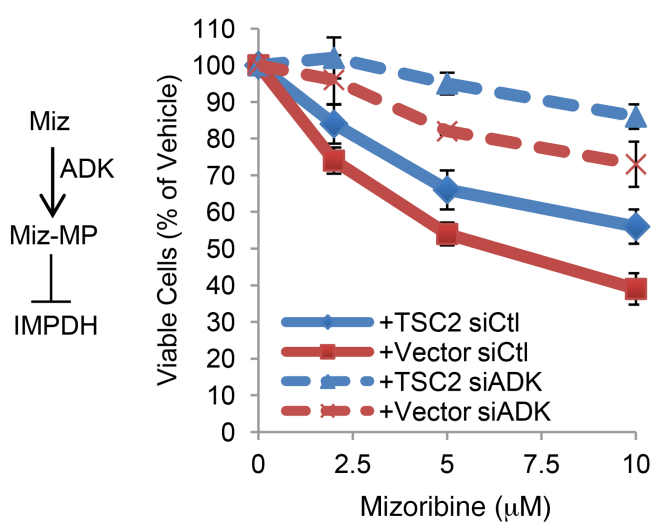

E

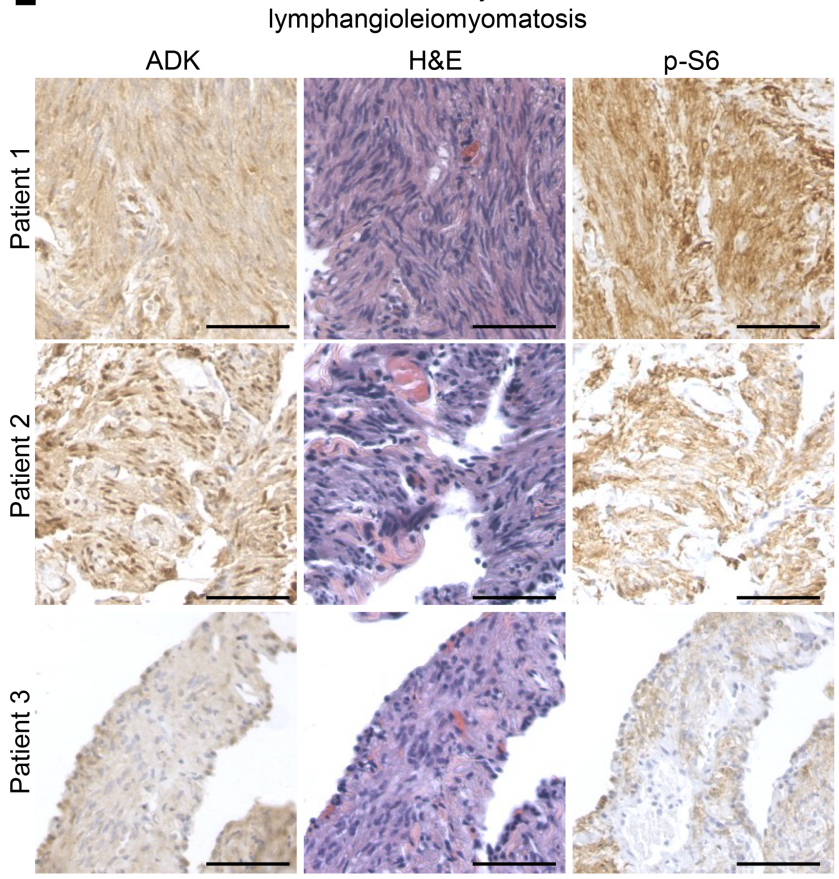

C

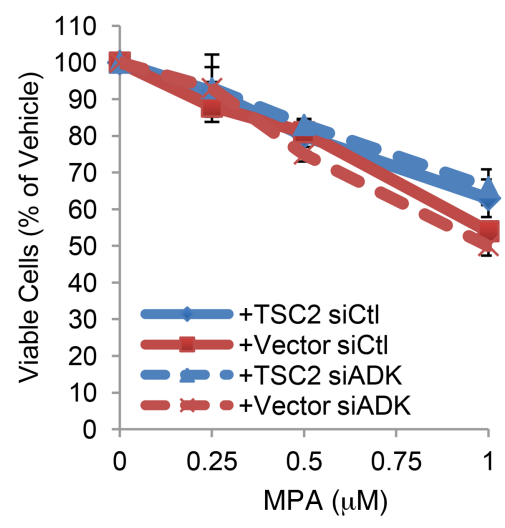

D SiRNA: $\frac{+\mathrm{TSC} 2}{\mathrm{Ctl} \text { ADK }} \frac{+\mathrm{Vector}}{\mathrm{Ctl} \text { ADK }}$

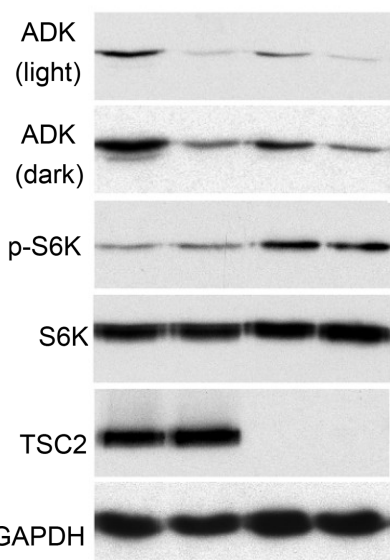

F

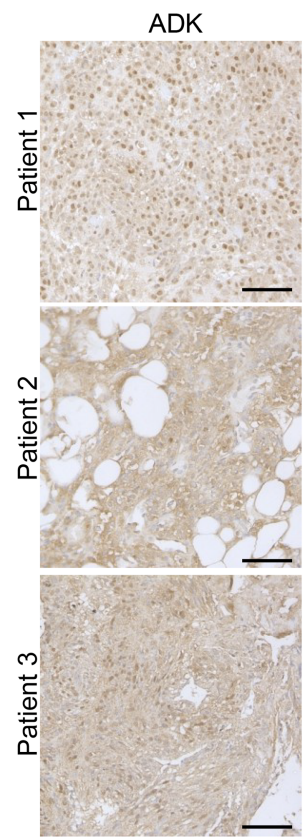

Renal angiomyolipoma

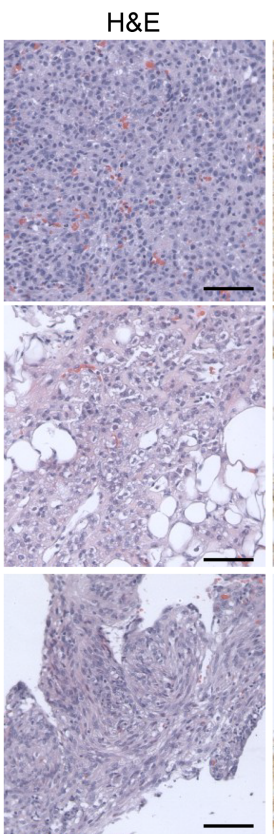

p-S6

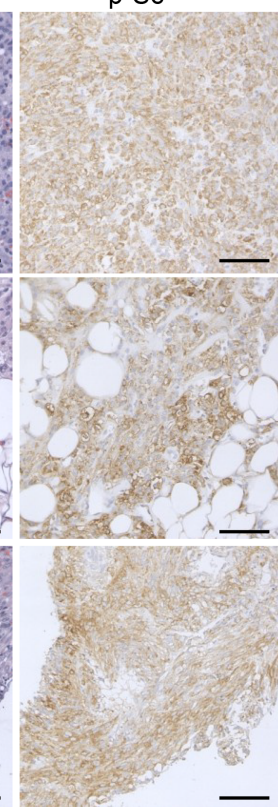

Figure 8. ADK, required for mizoribine action, is expressed in human TSC-associated pulmonary LAM and renal angiomyolipoma. (A) Mizoribine is phosphorylated by ADK to produce mizoribine monophosphate (Miz-MP), the product that inhibits IMPDH. (B-D) 105K cells stably reconstituted with empty vector or wild-type TSC2 were transfected with control (siCtl) or ADK-targeting siRNAs (siADK) and treated for 48 hours with indicated concentrations of (B) mizoribine or (C) MPA. Viable cells were quantified by CellTiter-Glo and graphed as percentage of vehicle-treated cells. $n=3$ independent experiments. (D) Immunoblots showing ADK knockdown and TSC2 reconstitution efficiency. Results are from a single experiment done alongside a replicate in panels B and $\mathbf{C}$. (E and $\mathbf{F})$ H\&E and IHC staining of (E) 3 human pulmonary LAM and (F) 3 renal angiomyolipoma specimens from LAM and TSC patients. Graphical data are presented as mean of indicated replicates \pm SEM. Scale bars: $100 \mu \mathrm{m}$.

Adenosine kinase, required for mizoribine action, is expressed in human TSC-associated lesions. To inhibit $\mathrm{IMPDH}$, the purine nucleoside analog mizoribine must be phosphorylated by adenosine kinase (ADK) to produce mizoribine monophosphate (Figure 8A) $(18,39)$. siRNA-mediated knockdown of ADK in Tsc2-deficient $105 \mathrm{~K}$ tumor cells reconstituted with either empty vector or wild-type TSC2 confirmed that $\mathrm{ADK}$ is required for the cell growth-inhibitory effects of mizoribine, but not MPA (Figure 8, B and C), with remaining mizoribine activity reflecting incomplete knockdown of ADK (Figure 8D). We previously found, and recapitulate in this study, that the human $\mathrm{TSC2}^{-1-}$ renal angiomyolipoma-derived 621-101 cell line is highly resistant to mizoribine, regardless of TSC2 status (Supplemental Figure 1I), and this resistance is due to very low expression of ADK (18). If mizoribine is to be repurposed for TSC-associated pathologies, ADK expression would be required in TSC tumor cells, which we examined in a panel of 
archived LAM and TSC patient samples. IHC staining confirmed ADK expression localized to both the cytosol and nucleus (as reported previously in ref. 40) in both pulmonary LAM and TSC-associated renal angiomyolipoma patient samples. ADK staining was concentrated in the distinct smooth muscle-like cells with spindle morphology that are characteristic of these lesions, which also stained strongly for ribosomal S6 phosphorylation in adjacent serial sections (Figure 8, E and F). Thus, these lesions and other TSC-associated tumors may be candidates for mizoribine therapy.

\section{Discussion}

We previously found that uncontrolled mTORC1 activation increases dependence on IMPDH for cell and tumor growth and viability by increasing the cellular demand for guanylate nucleotides required to support rRNA synthesis as part of mTORC1-driven ribosome biogenesis (18). Here we directly compared clinically approved IMPDH inhibitors and find that mizoribine is a superior antitumor agent compared with mycophenolate in TSC2-deficient tumors, which are driven by robust mTORC1 activation. Collectively, we observe strong antitumor effects of mizoribine compared with modest effects of mycophenolate in 4 TSC2deficient tumor models: $105 \mathrm{~K}$ and ELT3 xenograft tumors in immunocompromised mice (Figure 3 and Figure 4), syngeneic 105K xenograft tumors in immunocompetent mice (Figure 6 and Figure 7), and sporadic renal tumors in $T s c 2^{+/-}$mice (Supplemental Figure 5I and ref. 18). The greater efficacy of mizoribine is observed despite remarkable similarity to $\mathrm{MMF}$ in biological effects on immune cells, as established in both humans and animal models $(29,41-43)$ and confirmed in our study (Figure 6B). These preclinical studies suggest mizoribine could be an effective treatment in tumors with active mTORC1, including those in patients with TSC. Mizoribine has a potential advantage over rapamycin and its analogs, which are often used for treating TSC and LAM lesions, in its ability to induce tumor cell death and correspondingly slow the rate of tumor regrowth if treatment is discontinued. Importantly, decades of clinical use of mizoribine indicate that it has a safety profile in humans that is similar or favorable to mycophenolate and rapamycin $(23,42,44,45)$.

It is important to note that tumors lacking ADK expression would likely be an exception to the findings presented here, in which case MMF might have an advantage over mizoribine due to the inability of the tumor cells to convert mizoribine to the active mizoribine monophosphate. Thus, we confirmed ADK expression in human TSC-associated LAM and renal angiomyolipoma samples. We also identified accumulation of the purine synthesis intermediate AICAR as a metabolic biomarker of mizoribine efficacy in cells, tumors, and normal tissues and plasma, which could be used to monitor the biological activity of mizoribine.

Although mizoribine and MPA both inhibit IMPDH and are used for similar clinical indications, we find that they have differential effects on signaling and metabolism in vivo. The greater antitumor efficacy of mizoribine compared with MMF likely results from a greater depletion of tumor guanylate nucleotides in response to mizoribine (Figure 5C). Unlike mizoribine, MMF was found to partially inhibit mTORC1 signaling, possibly resulting from the partial depletion of tumor adenylates, which are sensed by mTORC1 and required for its activity (27). mTORC1 inhibition could limit the rate of guanylate nucleotide depletion, and thus the antitumor efficacy of MMF, by reducing rRNA synthesis and thus nucleotide demand. Indeed, our previous studies and Supplemental Figure 2G demonstrate that selective sensitivity to IMPDH inhibitors requires robust and sustained mTORC1 activation, with consequent elevated rates of rRNA synthesis (18). It is also possible that inhibitory effects on mTORC1 signaling could underlie the lack of AICAR accumulation in mice treated with MMF, and we have previously shown that cotreatment with rapamycin attenuates the mizoribine-induced increase in tumor AICAR levels, reflecting a reduction in mTORC1-driven flux through the de novo purine synthesis pathway $(4,18)$.

It is remarkable that available inhibitors of other essential enzymes in the de novo or salvage nucleotide synthesis pathways, including several compounds in clinical use, failed to show the selective inhibitory effects on growth and viability of TSC2-deficient cells that we observed with IMPDH inhibitors. These results suggest that targeting guanylate synthesis, specifically, offers a unique ability to exploit the increased demand for nucleotides that comes with mTORC1-driven ribosomal RNA synthesis. Guanylate nucleotides are enriched in ribosomal RNA, accounting for 34\% of nucleotides in the mature mammalian ribosome and $37 \%$ of nucleotides in the $45 \mathrm{~S}$ pre-rRNA that is processed into the mature $18 \mathrm{~S}, 5.8 \mathrm{~S}$, and $28 \mathrm{~S}$ rRNAs. Correspondingly, the rate of incorporation of available GTP into rRNA is greater than ATP, UTP, or CTP (20). Free intracellular guanylates are maintained at substantially lower concentrations than adenylates and may also be lower than pyrimidines in some cell types (46-50). Thus, lower basal levels combined with greater enrichment in rRNA could underlie the distinct importance of guanylate synthesis in 
cells with elevated rates of rRNA synthesis and provide a mechanism for the selective vulnerability of cells with elevated mTORC1 signaling to IMPDH inhibitors, relative to other inhibitors of nucleotide synthesis.

It is worth noting that, like mTORC1, the oncogene c-Myc drives both ribosome biogenesis and de novo nucleotide synthesis $(51,52)$. Dependence on IMPDH correlates with Myc expression in small-cell lung cancer, where mizoribine demonstrated antitumor efficacy in preclinical tumor models (19). IMPDH dependence correlating with a high rate of rRNA synthesis was also recently demonstrated in glioblastoma, where xenograft tumor growth was slowed by MMF and completely blocked by genetic knockout of IMPDH1 and IMPDH2 (20). MMF has also demonstrated efficacy in xenograft tumors established using other human cancer cell lines $(21,22)$. This growing body of evidence suggests that IMPDH inhibitors could potentially be repurposed as antitumor agents, and our study indicates that mizoribine should be considered a leading candidate among these drugs.

\section{Methods}

Cell lines and culture conditions. Tsc2 $2^{+/+}$Trp $53^{-/-}$and Tsc2 $2^{-1-}$ Trp $53^{-/-}$MEFs were provided by David Kwiatkowski (Harvard Medical School, Boston, Massachusetts, USA) and were described previously (53). Tsc2 ${ }^{-1} 105 \mathrm{Ks}$ stably expressing empty vector or TSC2 (54), 621-101 cells stably expressing empty vector or TSC2 (55), and HeLa cells stably expressing shRNAs targeting luciferase or TSC2 (56) were described previously. Tsc2 ${ }^{-1-}$ ELT3 cells were provided by Cheryl Walker (Baylor College of Medicine, Houston, Texas, USA) and were described previously (57). Cells were grown in DMEM (VWR, Avantor, 45000-312) plus 10\% heat-inactivated fetal bovine serum (Thermo Fisher Scientific 10437-028) and 1\% penicillin/streptomycin (Corning 30-002-Cl).

Chemical compounds. Where indicated, the following compounds were added into the cell culture medium at final concentrations stated in the figures and legends: mizoribine (Selleckchem S1384 and MilliporeSigma M3047), MPA (MilliporeSigma M3536), MMF (Selleckchem S1501), ribavirin (MilliporeSigma R9644), AVN-944 (Chemietek CT-AVN944), rapamycin (MilliporeSigma 53123-88-9), methotrexate (MilliporeSigma A6770), 6-mercaptopurine (MilliporeSigma 852678), A771726 (Calbiochem 100128), brequinar (MilliporeSigma SML0113), pyrazofurin (MilliporeSigma SML1502), 3-deazauridine (Santa Cruz Biotechnology sc-394445), 5-fluorouracil (Tocris 3257), and guanosine (MilliporeSigma G6752).

siRNA transfections. Control nontargeting siRNA (GE Dharmacon D-001810-10-50) or siRNA targeting ADK (GE Dharmacon L-062728-00-0005) were transfected at $12.5 \mathrm{nM}$ final concentration using Lipofectamine RNAiMAX (Thermo Fisher Scientific 13778150) according to the manufacturer's instructions.

Cell viability assays. Cell viability was determined by trypan blue (MilliporeSigma T8154) exclusion and quantified using a hemocytometer. Where indicated, viable cell counts were measured using the CellTiter-Glo Luminescent Cell Viability Assay (Promega G7573) according to the manufacturer's instructions. Annexin V/PI staining was performed using the Dead Cell Apoptosis Kit (Thermo Fisher Scientific V13245), according to the manufacturer's instructions. Staining was measured with a BD LSRFortessa flow cytometer and analyzed with FlowJo version 10.2 software.

Cell cycle analysis. Cell cycle analysis was performed using the Thermo Fisher Scientific FxCycle Violet Staining Kit (F10347) according to the manufacturer's instructions. Staining was measured with a BD LSRFortessa flow cytometer and analyzed using FlowJo version 10.2 software.

Immunoblotting and antibodies. Cells were lysed in buffer containing $20 \mathrm{mM}$ Tris pH 7.5, $140 \mathrm{mM} \mathrm{NaCl}$, $1 \mathrm{mM}$ EDTA, 10\% glycerol, 1\% Triton X-100, 1 mM DTT, $50 \mathrm{mM} \mathrm{NaF,} \mathrm{protease} \mathrm{inhibitor} \mathrm{cocktail} \mathrm{(Milli-}$ poreSigma P8340), and phosphatase inhibitor cocktails 2 (MilliporeSigma P5726) and 3 (MilliporeSigma P0044) used at 1:100. Western blots were performed using the following antibodies at 1:1000 dilution unless otherwise indicated: phospho-p70 S6 kinase T389 (Cell Signaling Technology [CST] 9234 clone 108D2), p70 S6 kinase (CST 2708 clone 49D7), TSC2 (CST 4308 clone D93F12 and CST 3612), Rheb (CST 13879 clone E1G1R), GAPDH (CST 5174 clone D16H11), cleaved caspase-3 D175 (CST 9664 clone 5A1E), ADK (Santa Cruz Biotechnology sc-514588 clone H-1, 1:500), phospho-Chk1 S345 (CST 2348 clone 133D3), Chk1 (CST 2360 clone 2G1D5), phospho-histone H2A.X S139 (CST 9718 clone 20E3), histone H2A.X (CST 7631 clone D17A3), Bak (CST 12105 clone D4E4), Bax (CST 2772), Bim (CST 2933 clone C34C5), Bc12 (Abcam ab182858 clone EPR17509), Mcl1 (CST 5453 clone D35A5), Bcl-xl (CST 2764 clone 54H6), c-Myc (CST 9402), and actin (GeneTex GTX629630 clone GT5512, 1:5000).

Mouse studies. All animal procedures were approved by the Harvard Institutional Animal Care and Use Committee. For xenograft tumor studies, 2.5 million 105K cells or ELT3 cells expressing luciferase (58) were subcutaneously injected in a 1:1 mixture with Matrigel (BD 356237) into the flank of 6- to 
7-week-old NSG mice (The Jackson Laboratory 005557) or C57BL/6J mice (The Jackson Laboratory 000664). Treatment with mizoribine (Selleckchem S1384), rapamycin (LC Laboratories R-5000), or MMF (MilliporeSigma 1448956) began 4 weeks later, when tumors first became palpable, using doses indicated in the figures and legends. Mice were assigned to treatment groups based on their tumor size so that the average tumor size per group was the same among all groups at the start of treatment. Tumor size was measured every 3 days using digital calipers. Tsc2 $2^{+/-}$mice on the A/J strain background were described previously $(59,60)$ and were treated through the TS Alliance Preclinical Consortium at PsychoGenics. Mice were randomly assigned for treatment with vehicle or MMF (75 mg/kg/d) by oral gavage for 1 month beginning at 7 months of age. Kidneys were resected, fixed in $4 \%$ paraformaldehyde for 24 hours, and embedded in paraffin. Kidneys were sectioned and tumor volume was quantified as previously described (59).

IHC. IHC staining was performed on formalin-fixed, paraffin-embedded tissue sections using a previously described staining protocol (18) with the following antibodies: phospho-S6 S235/236 (CST 4858 clone D57.2.2E 1:400), ADK (Abcam ab204430 1:20), Ki-67 (Abcam ab16667 clone SP6 1:100), and cleaved caspase-3 (CST 9664 clone 5A1E 1:100). Human pulmonary LAM and renal angiomyolipoma patient samples were collected according to an IRB protocol approved by Brigham and Women's Hospital.

Plasma collection from mice and blood cell counts. Blood was drawn by retro-orbital insertion of a heparinized microcapillary tube (Thermo Fisher Scientific 22-362-566), collected in EDTA-coated microtainer tubes (BD 365973), and centrifuged at $3000 \mathrm{~g}$ for 10 minutes at room temperature, and plasma was removed. Blood cell counts were determined using a Mascot HemaVet 950FS Hematology Analyzer.

Metabolite analyses by LC-MS/MS. Metabolites were extracted using $80 \%$ methanol and dried under nitrogen gas for targeted LC-MS/MS profiling via selected reaction monitoring (SRM) with polarity switching on a 6500 QTRAP mass spectrometer (AB/SCIEX) as previously described (5, 61, 62). For LC-MS/ MS analysis, metabolites were extracted from either $80 \mu \mathrm{L}$ plasma, $0.1-0.5 \mathrm{~g}$ tissue (with extraction buffer volume normalized to tissue weight), or one $10-\mathrm{cm}$ cell culture dish. Mizoribine was measured in negative ion mode using Q1/Q3 SRM transitions of 258.2/126 and MPA in positive ion mode using Q1/Q3 SRM transitions of 321.1/206.9. Data were analyzed by calculating the Q3 peak areas using MultiQuant 3.0 software (AB/SCIEX) and normalized to the median metabolite in each sample.

Statistics. Graphical data are presented as mean \pm SEM. $P$ values for pairwise comparisons were determined using an unpaired 2-tailed Student's $t$ test. A $P$ value less than 0.05 was considered significant. Statistical details for individual experiments can be found in their respective figure legends.

Study approval. All animal procedures were approved by the Harvard Institutional Animal Care and Use Committee. Human pulmonary LAM and renal angiomyolipoma patient samples were collected according to an IRB protocol approved by Brigham and Women's Hospital, and written informed consent was received from participants prior to inclusion in the study.

\section{Author contributions}

$\mathrm{AJV}$ and BDM conceptualized and designed experiments and wrote the manuscript, with editing by MCM and EPH. AJV, SKM, MCM, MET, and JMA conducted experiments and acquired data. AJV, JMA, EPH, and BDM analyzed data. EPH provided reagents.

\section{Acknowledgments}

We thank David J. Kwiatkowski, Gerta Hoxhaj, Issam Ben-Sahra, Hilaire Lam, and Min Yuan for helpful discussions and technical assistance. We also thank Steve Roberds, Dean Aguiar, and the TS Alliance Preclinical Consortium for experiments in $T s c 2^{+/-}$mice. This research was supported by grants from the National Institutes of Health (P01CA120964 to JMA, EPH, and BDM; P30CA006516 to JMA; and R35CA197459 to BDM) and a Rothberg Courage Award (194641) from the TS Alliance to BDM.

Address correspondence to: Alexander J. Valvezan or Brendan D. Manning, 665 Huntington Ave., SPH2 117, Boston, Massachusetts 02115, USA. Phone: 617.432.5614; Email: valvezan@hsph.harvard.edu (AJV); bmanning@hsph.harvard.edu (BDM). 
1. Ben-Sahra I, Manning BD. mTORC1 signaling and the metabolic control of cell growth. Curr Opin Cell Biol. 2017;45:72-82.

2. Menon S, Manning BD. Common corruption of the mTOR signaling network in human tumors. Oncogene. 2008;27(suppl 2):S43-S51.

3. Valvezan AJ, Manning BD. Molecular logic of mTORC1 signalling as a metabolic rheostat. Nat Metab. 2019;1(1):321-333.

4. Ben-Sahra I, Hoxhaj G, Ricoult SJH, Asara JM, Manning BD. mTORC1 induces purine synthesis through control of the mitochondrial tetrahydrofolate cycle. Science. 2016;351(6274):728-733.

5. Ben-Sahra I, Howell JJ, Asara JM, Manning BD. Stimulation of de novo pyrimidine synthesis by growth signaling through mTOR and S6K1. Science. 2013;339(6125):1323-1328.

6. Robitaille AM, et al. Quantitative phosphoproteomics reveal mTORC1 activates de novo pyrimidine synthesis. Science. 2013;339(6125):1320-1323.

7. Düvel K, et al. Activation of a metabolic gene regulatory network downstream of mTOR complex 1. Mol Cell. 2010;39(2):171-183.

8. Fruman DA, Rommel C. PI3K and cancer: lessons, challenges and opportunities. Nat Rev Drug Discov. 2014;13(2):140-156.

9. Hasbani DM, Crino PB. Tuberous sclerosis complex. Handb Clin Neurol. 2018;148:813-822.

10. Lam HC, Nijmeh J, Henske EP. New developments in the genetics and pathogenesis of tumours in tuberous sclerosis complex. J Pathol. 2017;241(2):219-225

11. Henske EP, Jóźwiak S, Kingswood JC, Sampson JR, Thiele EA. Tuberous sclerosis complex. Nat Rev Dis Primers. 2016;2:16035.

12. Gupta N, Henske EP. Pulmonary manifestations in tuberous sclerosis complex. Am J Med Genet C Semin Med Genet. 2018;178(3):326-337.

13. Bissler JJ, et al. Sirolimus for angiomyolipoma in tuberous sclerosis complex or lymphangioleiomyomatosis. $N$ Engl J Med. 2008;358(2):140-151.

14. Bissler JJ, et al. Angiomyolipoma rebound tumor growth after discontinuation of everolimus in patients with tuberous sclerosis complex or sporadic lymphangioleiomyomatosis. PLoS ONE. 2018;13(9):e0201005.

15. Huynh $\mathrm{H}$, et al. Loss of tuberous sclerosis complex 2 (TSC2) is frequent in hepatocellular carcinoma and predicts response to mTORC1 inhibitor everolimus. Mol Cancer Ther. 2015;14(5):1224-1235.

16. Guo Y, et al. TSC1 involvement in bladder cancer: diverse effects and therapeutic implications. J Pathol. 2013;230(1):17-27.

17. Ilagan E, Manning BD. Emerging role of mTOR in the response to cancer therapeutics. Trends Cancer. 2016;2(5):241-251.

18. Valvezan AJ, et al. mTORC1 couples nucleotide synthesis to nucleotide demand resulting in a targetable metabolic vulnerability. Cancer Cell. 2017;32(5):624-638.e5.

19. Huang F, et al. Inosine monophosphate dehydrogenase dependence in a subset of small cell lung cancers. Cell Metab. 2018;28(3):369-382.e5.

20. Kofuji S, et al. IMP dehydrogenase-2 drives aberrant nucleolar activity and promotes tumorigenesis in glioblastoma. Nat Cell Biol. 2019;21(8):1003-1014.

21. Domhan S, et al. Molecular mechanisms of the antiangiogenic and antitumor effects of mycophenolic acid. Mol Cancer Ther 2008;7(6):1656-1668.

22. Tressler RJ, Garvin LJ, Slate DL. Antitumor activity of mycophenolate mofetil against human and mouse tumors in vivo. Int $J$ Cancer. 1994;57(4):568-573.

23. Ishida $\mathrm{H}$, et al. A prospective randomized, comparative trial of high-dose mizoribine versus mycophenolate mofetil in combination with tacrolimus and basiliximab for living donor renal transplant: a multicenter trial. Exp Clin Transplant. 2016;14(5):518-525.

24. Thomas E, Ghany MG, Liang TJ. The application and mechanism of action of ribavirin in therapy of hepatitis C. Antivir Chem Chemother. 2012;23(1):1-12.

25. Te HS, Randall G, Jensen DM. Mechanism of action of ribavirin in the treatment of chronic hepatitis C. Gastroenterol Hepatol (N Y). 2007;3(3):218-225.

26. Floryk D, Thompson TC. Antiproliferative effects of AVN944, a novel inosine 5-monophosphate dehydrogenase inhibitor, in prostate cancer cells. Int J Cancer. 2008;123(10):2294-2302.

27. Hoxhaj G, et al. The mTORC1 signaling network senses changes in cellular purine nucleotide levels. Cell Rep. 2017;21(5):1331-1346.

28. Emmanuel N, et al. Purine nucleotide availability regulates mTORC1 activity through the Rheb GTPase. Cell Rep. 2017;19(13):2665-2680.

29. Ishikawa H. Mizoribine and mycophenolate mofetil. Curr Med Chem. 1999;6(7):575-597.

30. Kalluri HV, Hardinger KL. Current state of renal transplant immunosuppression: present and future. World J Transplant. 2012;2(4):51-68.

31. Liu HJ, et al. TSC2-deficient tumors have evidence of T cell exhaustion and respond to anti-PD-1/anti-CTLA-4 immunotherapy. JCI Insight. 2018;3(8):98674.

32. Stypinski D, Obaidi M, Combs M, Weber M, Stewart AJ, Ishikawa H. Safety, tolerability and pharmacokinetics of higher-dose mizoribine in healthy male volunteers. Br J Clin Pharmacol. 2007;63(4):459-468.

33. Takada K, et al. Pharmacokinetics of bredinin in renal transplant patients. Eur J Clin Pharmacol. 1983;24(4):457-461.

34. de Winter BC, et al. Population pharmacokinetics of mycophenolic acid : a comparison between enteric-coated mycophenolate sodium and mycophenolate mofetil in renal transplant recipients. Clin Pharmacokinet. 2008;47(12):827-838.

35. Romano $P$, et al. Longitudinal pharmacokinetics of mycophenolic acid in elderly renal transplant recipients compared to a younger control group: data from the nEverOld trial. Eur J Drug Metab Pharmacokinet. 2019;44(2):189-199.

36. Tate PM, Mastrodomenico V, Mounce BC. Ribavirin induces polyamine depletion via nucleotide depletion to limit virus replication. Cell Rep. 2019;28(10):2620-2633.e4.

37. Marinello E, Pizzichini M, Di Stefano A, Terzuoli L, Pagani R. Purine nucleotide synthesis in rat liver after castration. Adv Exp Med Biol. 1991;309B:371-374.

38. Fustin JM, Doi M, Yamada H, Komatsu R, Shimba S, Okamura H. Rhythmic nucleotide synthesis in the liver: temporal segregation of metabolites. Cell Rep. 2012;1(4):341-349.

39. Koyama H, Tsuji M. Genetic and biochemical studies on the activation and cytotoxic mechanism of bredinin, a potent inhibitor of purine biosynthesis in mammalian cells. Biochem Pharmacol. 1983;32(23):3547-3553.

40. Boison D. Adenosine kinase: exploitation for therapeutic gain. Pharmacol Rev. 2013;65(3):906-943.

41. Xing S, Yang J, Zhang X, Zhou P. Comparative efficacy and safety of mizoribine with mycophenolate mofetil for Asian renal 
transplantation--a meta-analysis. Clin Biochem. 2014;47(7-8):663-669.

42. Feng X, et al. Mizoribine versus mycophenolate mofetil or intravenous cyclophosphamide for induction treatment of active lupus nephritis. Chin Med J. 2014;127(21):3718-3723.

43. Shimmura H, Tanabe K, Habiro K, Abe R, Toma H. Combination effect of mycophenolate mofetil with mizoribine on cell proliferation assays and in a mouse heart transplantation model. Transplantation. 2006;82(2):175-179.

44. Zhang X, Fu S, Han S, Zheng X, Wang L. The argument for the use of mizoribine in renal transplantation: a meta-analysis and systemic review. Transpl Immunol. 2013;28(2-3):106-111.

45. Ju MK, et al. Mizoribine versus mycophenolate mofetil in combination therapy with tacrolimus for de novo kidney transplantation: evaluation of efficacy and safety. Transplant Proc. 2013;45(4):1481-1486.

46. Park JO, et al. Metabolite concentrations, fluxes and free energies imply efficient enzyme usage. Nat Chem Biol. 2016;12(7):482-489.

47. Guo JY, et al. Autophagy provides metabolic substrates to maintain energy charge and nucleotide pools in Ras-driven lung cancer cells. Genes Dev. 2016;30(15):1704-1717.

48. Ferraro P, Franzolin E, Pontarin G, Reichard P, Bianchi V. Quantitation of cellular deoxynucleoside triphosphates. Nucleic Acids Res. 2010;38(6):e85

49. Wilson PM, Labonte MJ, Russell J, Louie S, Ghobrial AA, Ladner RD. A novel fluorescence-based assay for the rapid detection and quantification of cellular deoxyribonucleoside triphosphates. Nucleic Acids Res. 2011;39(17):e112.

50. Traut TW. Physiological concentrations of purines and pyrimidines. Mol Cell Biochem. 1994;140(1):1-22.

51. Liu YC, et al. Global regulation of nucleotide biosynthetic genes by c-Myc. PLoS One. 2008;3(7):e2722.

52. Stine ZE, Walton ZE, Altman BJ, Hsieh AL, Dang CV. MYC, metabolism, and cancer. Cancer Discov. 2015;5(10):1024-1039.

53. Zhang $\mathrm{H}$, et al. Loss of Tsc1/Tsc2 activates mTOR and disrupts PI3K-Akt signaling through downregulation of PDGFR. JClin Invest. 2003;112(8):1223-1233.

54. Filippakis H, et al. Lysosomal regulation of cholesterol homeostasis in tuberous sclerosis complex is mediated via NPC1 and LDL-R. Oncotarget. 2017;8(24):38099-38112.

55. Yu J, Astrinidis A, Howard S, Henske EP. Estradiol and tamoxifen stimulate LAM-associated angiomyolipoma cell growth and activate both genomic and nongenomic signaling pathways. Am J Physiol Lung Cell Mol Physiol. 2004;286(4):L694-L700

56. Huang J, Dibble CC, Matsuzaki M, Manning BD. The TSC1-TSC2 complex is required for proper activation of mTOR complex 2. Mol Cell Biol. 2008;28(12):4104-4115.

57. Hodges LC, et al. Transdominant suppression of estrogen receptor signaling by progesterone receptor ligands in uterine leiomyoma cells. Mol Cell Endocrinol. 2002;196(1-2):11-20.

58. Yu JJ, et al. Estrogen promotes the survival and pulmonary metastasis of tuberin-null cells. Proc Natl Acad Sci U S A. 2009;106(8):2635-2640.

59. Auricchio N, Malinowska I, Shaw R, Manning BD, Kwiatkowski DJ. Therapeutic trial of metformin and bortezomib in a mouse model of tuberous sclerosis complex (TSC). PLoS One. 2012;7(2):e31900

60. Guo Y, Kwiatkowski DJ. Equivalent benefit of rapamycin and a potent mTOR ATP-competitive inhibitor, MLN0128 (INK128), in a mouse model of tuberous sclerosis. Mol Cancer Res. 2013;11(5):467-473

61. Yuan M, et al. Ex vivo and in vivo stable isotope labelling of central carbon metabolism and related pathways with analysis by LC-MS/MS. Nat Protoc. 2019;14(2):313-330.

62. Yuan M, Breitkopf SB, Yang X, Asara JM. A positive/negative ion-switching, targeted mass spectrometry-based metabolomics platform for bodily fluids, cells, and fresh and fixed tissue. Nat Protoc. 2012;7(5):872-881. 